\title{
Title: Saturn's magnetic field revealed by the Cassini Grand Finale
}

Authors: Michele K. Dougherty ${ }^{1 *}$, Hao Cao ${ }^{2,3,1}$, Krishan K. Khurana ${ }^{4}$, Gregory J. Hunt ${ }^{1}$, Gabrielle Provan $^{5}$, Stephen Kellock ${ }^{1}$, Marcia E. Burton ${ }^{6}$, Thomas A. Burk ${ }^{6}$, Emma J. Bunce ${ }^{5}$, Stanley W.

5 H. Cowley ${ }^{5}$, Margaret G. Kivelson ${ }^{4,7}$, Christopher T. Russell ${ }^{4}$, David J. Southwood ${ }^{1}$.

\section{Affiliations:}

${ }^{1}$ Physics Department, The Blackett Laboratory, Imperial College London, SW7 2AZ, UK.

${ }^{2}$ Department of Earth and Planetary Sciences, Harvard University, 20 Oxford Street, Cambridge, 10 MA 02138, USA.

${ }^{3}$ Division of Geological and Planetary Sciences, California Institute of Technology, $1200 \mathrm{E}$ California Blvd, Pasadena, CA 91125, USA.

${ }^{4}$ Department of Earth, Planetary, and Space Sciences, University of California, Los Angeles, 90025, USA.

155 Department of Physics and Astronomy, University of Leicester, Leicester, LE1 7RH, UK.

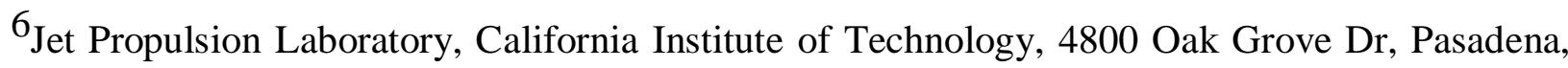
CA 91109, USA. 
${ }^{7}$ Department of Climate and Space Sciences and Engineering, University of Michigan, Ann Arbor, MI 40109, USA.

${ }^{*}$ To whom correspondence should be addressed;

$5 \quad$ E-mail: m.dougherty@imperial.ac.uk

\begin{abstract}
During 2017, the Cassini fluxgate magnetometer made in-situ measurements of Saturn's magnetic field at distances $\sim 2550 \pm 1290$ kilometers above the 1 bar surface along twenty-two highly inclined Grand Finale orbits. These observations confirm an extreme level of axisymmetry of Saturn's internal magnetic field and displacement of the magnetic equator northwards from the planet's physical equator. Persistent small-scale magnetic structures corresponding to high-degree (>3) axisymmetric magnetic moments were observed, suggesting secondary shallow dynamo action in the semi-conducting region of Saturn's interior. Some high-degree magnetic moments could arise from strong high-latitude concentrations of magnetic flux within the planet's deep dynamo. A strong field-aligned current (FAC) system is located between Saturn and the inner edge of its D-ring, with strength comparable to the high-latitude auroral FACs.
\end{abstract}

One Sentence Summary: Magnetometer measurements during Cassini Grand Finale revealed a new field-aligned current system between Saturn and the rings, high-degree axisymmetric internal magnetic moments suggesting a secondary dynamo action in the semi-conducting region of Saturn. 


\section{Main Text:}

The internal magnetic field of a planet provides a window into its interior (1). Saturn's magnetic field was first measured by the Pioneer 11 magnetometers (2,3) and shortly afterwards by Voyagers 1 and $2(4,5)$. Those observations $(2-7)$ revealed that Saturn's magnetic axis is tilted with respect to the planetary spin-axis by less than $1^{\circ}$. The axisymmetric part of Saturn's internal field is more complex than a dipole (commonly denoted with the Gauss coefficient $g_{1}{ }^{0}$ ), with the axisymmetric quadrupole and octupole magnetic moment contributions (coefficients $g_{2}{ }^{O}$ and $g_{3}{ }^{O}$ respectively) amounting to about $10 \%$ of the dipole moment contribution when evaluated at the 1 10 bar surface of the planet. Three decades later, Cassini magnetometer (MAG) measurements $(8-12)$ made prior to the Grand Finale phase refined Saturn's dipole tilt to less than $0.06^{\circ}(11)$. Secular variation is at least an order of magnitude slower than at Earth (11), and magnetic flux is expelled from the equatorial region towards mid-to-high latitude (12). Magnetic moments beyond spherical harmonic degree 3, with Gauss coefficients on the order of 100 nano-Tesla $(n T)$, were suggested

15 by the MAG measurements during Cassini Saturn Orbit Insertion (SOI) in 2004 (12), but were not well resolved due to the limited spatial coverage of SOI, with periapsis (closest approach) at 1.33 $R_{S}$ (where $1 R_{S}=60,268 \mathrm{~km}$ is the 1-bar equatorial radius of Saturn) and latitudinal coverage within $\pm 20^{\circ}(12)$.

20 Saturn's main magnetic field is believed to be generated by rotating convective motion in the metallic hydrogen $(13,14)$ layer of Saturn through the magnetohydrodynamic (MHD) dynamo process $(1,15-17)$. The highly symmetric nature of Saturn's internal magnetic field with respect 
to the spin-axis not only defies an accurate determination of the rotation rate of Saturn's deep interior but also challenges our understanding of the MHD dynamo process, since the Cowling's theorem (18) precludes the possibility of maintaining a perfectly axisymmetric magnetic field through an active MHD dynamo. The possibility that we are observing Saturn's magnetic field at a time with no active dynamo action can be safely ruled out given the sizable quadrupole and octupole magnetic moments, since these would decay with time at a much faster rate than the dipole (see $\mathrm{Ch} 2$ of 19). In addition to the main dynamo action in the metallic hydrogen layer, deep zonal flow (differential rotation) and small-scale convective motion in the semi-conducting region of Saturn could lead to a secondary dynamo action as recently proposed by (20).

We analyze magnetometer measurements made during the last phase of the Cassini mission. The Cassini Grand Finale phase began on 26 April 2017, with the spacecraft diving through the gap between Saturn's atmosphere and the inner edge of its D-ring 22 times, making a final dive into the atmosphere on 15 September 2017. Periapses were $\sim 2550 \pm 1290 \mathrm{~km}$ above the atmosphere's

15 one bar level, equivalent to $\sim 1.04 \pm 0.02 R_{S}$ from the center of Saturn (Fig. S1). The low periapses, high inclination $\left(\sim 62^{\circ}\right)$ and proximity to the noon-midnight meridian in local time provided an opportunity to investigate Saturn's internal magnetic field and the magnetic environment between Saturn and its rings (see Figures S1 \& S2). The fluxgate magnetometer (8) made continuous (32 measurements per second) three-component magnetic field measurements until the final second of spacecraft transmission. For analysis, we averaged the data to 1 second resolution. During $\sim \pm 50$ mins around periapsis, the instrument used its highest dynamic range mode, which can measure magnetic field components up to 44,000 $n T$ with digitization noise of $5.4 n T$ (8). This mode had not been used since an Earth fly-by in August 1999 (21) and therefore required recalibration during 
the Grand Finale orbits. Four spacecraft roll campaigns and numerous spacecraft turns enabled this recalibration. The spacecraft attitude needed to be known to a high accuracy of 0.25 milliradians, but intermittent suspension of the spacecraft star identification system during phases of instrument targeting resulted in gaps in the required information. These gaps were filled by reconstructing spacecraft attitude using information from the onboard gyroscopes. Attitude reconstruction is currently available for 9 of the first 10 orbits (see Table S1 for details), so we restrict our analysis to those orbits. Individual orbits of Cassini are commonly referred to using a Rev number; these 9 orbits are Rev 271 to 280 excluding Rev 277.

\section{External magnetic field from sources in the magnetosphere and ionosphere of Saturn}

In deriving the internal planetary magnetic field from in-situ MAG measurements, it is necessary to separate the internal planetary field from exterior sources of field (i.e. currents in the magnetosphere and ionosphere). In the Saturnian system, the simplest external source to correct is the magnetodisk current, which flows in the equatorial region far out in the magnetosphere (2226). Like the terrestrial ring current, the magnetodisk current produces a fairly uniform depression in the field strength close to the planet and is normally treated as axisymmetric (see Figure S3). At Saturn, the magnetodisk current contributes about $15 n T$ of magnetic field parallel to the spin-axis of Saturn $\left(B_{Z}\right)$ in the inner magnetosphere (22-26). Magnetopause and magnetotail currents, which determine the properties of the outer magnetosphere, contribute about $2 n T B Z$ field in the inner magnetosphere of Saturn $(22,26)$. More complex are the ubiquitous planetary period oscillations (PPOs), global 10.6 to 10.8 hour oscillations, at close to the expected planetary rotation period that are present in all Saturn magnetospheric parameters (see 27, 28 for reviews). The PPOs arise from a rotating electrical current systems with sources and sinks in Saturn's ionosphere and 
magnetosphere and closure currents that flow along magnetic field lines (commonly referred to as field-aligned currents or FACs) and map to the auroral regions. Normally two periods are present at any one time, with one dominant in the northern hemisphere and the other in the southern (2933), which during the interval studied here were 10.79 and $10.68 \mathrm{~h}$, respectively (33). The rotation periods, amplitudes and relative amplitudes of the two PPO systems vary slowly over Saturn's seasons (34-36). These seasonal changes indicate that the source of the periods is most likely to be of atmospheric origin rather than deep within the planet $(37,38)$. Moreover, the PPO periods are a few percent longer compared to any likely rotation period of the deep interior of the planet (39-41). There is also a non-oscillatory system of strong field-aligned currents in the auroral region with current closure through the planetary ionosphere, and there are distributed field-aligned currents at other latitudes (42-44). This non-oscillatory current system is associated with magnetospheric plasma that rotates more slowly than the planet (42). During the Grand Finale phase, the Cassini S/C was close enough to the planet during periapse to detect directly currents in the ionosphere. We describe results on these external current systems first, as they must be 15 considered during analysis of the internal field.

Figure 1 shows the vector magnetic field measurements in IAU Saturn System III right-hand spherical polar coordinates with $r, \theta, \varphi$ denoting radial, meridional, and azimuthal directions respectively (45) from the nine Cassini Grand Finale orbits for which accurate spacecraft attitude 20 information is available. This coordinate system has its origin at the center of mass of Saturn, and the spin-axis of Saturn is the polar axis (zenith reference). The peak magnetic field strength encountered during these Grand Finale orbits is approximately 18,000 $n T$, well within the range of the fluxgate magnetometer (8). The peak field was not encountered during periapsis, but at mid- 
latitude in the southern hemisphere. Figure 1A-B show the dominant radial and meridional magnetic field components, $B_{r}$ and $B_{\theta}$, which exhibit similar behavior on each orbit. This repeatability, combined with the fixed local time of the orbits and the slight differences in orbital period, demonstrate that the planetary magnetic field is close to axisymmetric.

Figure $1 \mathrm{C}$ shows the azimuthal component of the field $B_{\varphi}$, which is almost 3 orders of magnitude smaller than the radial and meridional components outside the auroral field aligned currents region. If it originates from the interior of the planet, $B_{\varphi}$ would be expected to decay with radial distance and also be part of a non-axisymmetric field pattern (see Eq. 5). However, much of the observed $B_{\varphi}$ signal seems to be of external rather than internal origin. The magnetic perturbations from the northern and southern auroral currents through which the spacecraft flew are marked in Figure 1 and Figure 2. In Figure 2, the cylindrical coordinate system is adopted where $\rho$ is the perpendicular distance to the spin-axis of Saturn and $Z$ is the distance from the planetary equator of Saturn which is defined by its center of mass. These current sheets have been crossed many times during the 15 Cassini mission, but previous crossings occurred much further from the planet $(43,44,46,47)$. The sharp field gradients observed are due to local field aligned currents, and the short scale of the variation in the field indicates local current sources rather than relatively distant currents in the planet's interior. The scatter in the auroral field aligned currents from orbit to orbit is partly due to the variability of the aurora over time and partly due to the large contribution from the rotating PPO signals, which are encountered at different phases on each orbit. 
The narrow central peak in $B_{\varphi}$ ranging between 5-30 $n T$ observed along these Grand Finale orbits was unexpected. It can be seen from Fig. 1C that the large change in field slope takes place where the spacecraft crosses the magnetic field lines that map to the inner edge of the D-ring. Here we adopt $1.11 R_{S}$ from the center of Saturn in the equatorial plane as the D-ring inner edge. If the azimuthal field gradient is due to a local field-aligned current, the current within the D-ring is inter-hemispheric and always flows from the northern to the southern hemisphere along these nine Grand Finale orbits. There must be return currents either at lower altitude than the spacecraft penetrates in the ionosphere or at other local times. Assuming approximate axisymmetry (for azimuthal spatial scales much larger than latitudinal scales), Ampère's law combined with current continuity leads to a total interhemispheric current flow in the range 0.25-1.5 Million Ampere per radian of azimuth. Thus, this low latitude field-aligned current is comparable in strength to the currents observed on field lines connected to the high-latitude auroral region $(43,44,46,47)$. It thus may be part of a global magnetospheric-ionospheric coupling system. Alternatively, the system might be purely of ionospheric origin, in which case it could be due to skewed flow between 15 northern and southern ends of the field line (48). All the orbit periapses are near local noon, so the repeatability from periapsis to periapsis does not rule out a dependency on local time.

There is a clear difference between the signatures of northern and southern auroral field-aligned currents and again magnetic mapping reveals the source. The locations of the auroral current sheets 20 are well-known magnetically from previous Cassini measurements $(43,44,46)$, see Figure 2. During Cassini's inbound trajectory in the north the spacecraft is located on the field lines just poleward of the main field-aligned currents region, as shown by the decreasing $B_{\varphi}$ which is indicative of the distributed downward current on polar field lines, as previously shown $(43,44,46)$. 
The less structured $B_{\varphi}$ during the outbound southern trajectories indicates that the southern hemisphere auroral field-aligned currents are not being fully crossed by the spacecraft, and instead we observe the center of field-aligned currents activity moving dynamically back and forth over the spacecraft.

\section{Saturn's internal magnetic field}

We now turn to analysis of the radial and meridional components of the measured magnetic field, which are dominated by the axisymmetric internal magnetic field. The highly inclined nature of the Grand Finale orbits allows direct determination close to the planet of Saturn's magnetic equator, defined as where the cylindrical radial component of the magnetic field, $B_{\rho}$, vanishes. Fig. 3a shows the measured $B_{\rho}$ as a function of the spacecraft's distance from the planetary equator of Saturn. The measured value along these orbits shows that Saturn's magnetic equator is $0.0466 \pm$ 0.0002 (1 standard deviation) $R_{S}$ northward of the planetary equator, measured at distance $\sim 1.05$ $R_{S}$ from the spin-axis of Saturn. This indicates a small yet non-negligible north-south asymmetry

in Saturn's magnetic field. Fig. 3b shows the distribution of the directly measured magnetic equator position of Saturn in the $\rho-Z$ plane and predictions from several pre-existing degree-3 models $(4,7,10,11)$. Several degree-3 internal field models have been derived (10) from different combinations of datasets; the two shown in Figure 3 were i) derived from data between Cassini SOI to July 2007 and ii) data from Cassini SOI to July 2007 combined with Pioneer 11, Voyager $1 \& 2$ data. A nominal magnetodisk field (see Methods) was added to the internal field when predicting the magnetic equator positions from existing models. A systematic decrease of the magnetic equator position with distance from the spin-axis is evident. This is a consequence of the axisymmetric part of the magnetic field, as illustrated by the predictions from existing 
axisymmetric models. In addition to this systematic trend, variations of measured magnetic equator positions at similar $\rho$ are also observed. These additional variations of the magnetic equator positions serve as a direct bound on possible non-axisymmetry of Saturn's internal magnetic field, because any internal non-axisymmetry would cause longitudinal variations of the magnetic equator positions. The measured peak to peak variation at similar cylindrical radial distances, $\sim 0.00030$ $R_{S}(18 \mathrm{~km})$, translates to a dipole tilt of $0.0095^{\circ}$, a guide value for possible internal nonaxisymmetry. This value is about one order of magnitude smaller than the upper limit on dipole tilt placed prior to the Grand Finale $(10-11,49)$. The corresponding field components at spacecraft altitude are only about $3 n T$ (in a background field of 18,000 $n T$ ), which is too small to be discerned from external fields in the measured $B_{\varphi}$. Such an extreme axisymmetric planetary magnetic field is difficult to explain with a dynamo model $(18,50-53)$.

Although the internal magnetic field of Saturn is highly axisymmetric (structure-less in the longitudinal direction), it exhibits latitudinal structures across many different length scales. This 15 is revealed by our retrieval of the axisymmetric Gauss coefficients of Saturn's internal magnetic field from these nine Grand Finale orbits. In this analysis, the magnetodisk field was explicitly included, adopting an analytical formula (25) with initial parameters taken from (22). The axisymmetric internal Gauss coefficients and the magnetodisk current field were then retrieved via an iterative process. To determine the internal Gauss coefficients, only measurements from range 203 of the fluxgate magnetometer were adopted $\left(|B|>10,000 n T, \mathrm{r}<1.58 R_{S}\right)$ and measurements from all nine orbits were treated as a single data set. These measurements were outside the auroral field aligned currents region, as shown in Figures 1 and 2. For retrieval of the magnetodisk current field, measurements from both range 3 and range 2 of the fluxgate magnetometer were utilised $(|B|$ 
$>400 n T, r<4.50 R S$ ), and each orbit was treated separately (see Table S2). Along the trajectory of the close-in portion of the Cassini Grand Finale orbits, the contributions from the magnetopause and magnetotail currents cannot be practically separated from the quasi-uniform magnetodisk current contribution, and thus are subsumed into our magnetodisk current model. The time-varying external (magnetodisk + magnetopause + magnetotail) $B_{Z}$ field could create a time-varying component of the internal dipole $g_{1}{ }^{O}$ through electromagnetic induction. The maximum magnetodisk field variation we have observed along the nine orbits is about $10 n T$, which, for an induction depth of $0.87 R_{S}$ (see Methods and Fig. S4), would induce a time variation in the internal dipole coefficient of about $3.3 n T$. This is less than the digitization level of the highest range of the 10 fluxgate magnetometer onboard Cassini $(5.4 n T)$, thus the detection of which is not straightforward.

We first investigated the minimum parameter set (in terms of internal Gauss coefficients) needed to adequately describe the observations, while applying no regularization to the parameters (see 15 Methods and ref. 54, 55). This experiment revealed that axisymmetric internal Gauss coefficients up to degree 9 are required to bring the root-mean-square (RMS) residual in range 3 below $10 n T$ (see Figure S5). Figure 4 shows that the residual $\left(B_{r}, B_{\theta}\right)$ after removal of the un-regularized degree 3 model (see Table S1 for coefficients) are on the order of $100 n T$, but have larger amplitude and larger spatial scales in the northern hemisphere compared to those in the southern hemisphere.

20 Figure 5 shows the residual $\left(B_{r}, B_{\theta}\right)$ as a function of latitude after removal of the un-regularized degree 6 model and the best-fitting magnetodisk model for each orbit (see Tables S1 and S2 for coefficients), which exhibits consistent small-scale features with amplitude $\sim 25 n T$ and typical spatial scale $\sim 25^{\circ}$ in latitude. The highly consistent nature (from orbit to orbit) of these small- 
scale magnetic perturbations in $\left(B_{r}, B_{\theta}\right)$, in contrast to the orbit-to-orbit varying $B_{\varphi}$ signature, suggests a source below the highly time variable currents in the ionosphere of Saturn.

Table 1 and Figure 6 present a new internal magnetic field model for Saturn, which we refer to as the Cassini 11 model with 11 denoting the highest spherical harmonic degree Gauss coefficient above the uncertainties (see Methods). Due to the fact that there was incomplete latitudinal coverage during the Grand Finale orbits which can lead to non-uniqueness in the solution, a regularized inversion technique $(54,55)$ has been employed to construct the Cassini 11 model. With the regularized inversion technique $(54,55)$, we explicitly seek internal field solutions that not only match the observations but also contain minimum magnetic power beyond degree 3 , when evaluated at a reference radius (see Methods). Table 1 presents the axisymmetric Gauss coefficients, five times the formal uncertainty ( $5 \sigma$, see Methods for definition), and the RMS residual of the Cassini 11 model. Figure 6 shows the resulting Mauersberger-Lowes (M-L) magnetic power spectrum (56-58) of the Cassini 11 model evaluated at the surface of Saturn. In

15 the Cassini 11 model, the axisymmetric Gauss coefficients between degree 4 and degree 11 are on the order of $10-100 n T$, while those above degree 11 are below the $5 \sigma$ uncertainty.

We have also investigated whether it is possible to obtain a simpler description of Saturn's internal magnetic field (in terms of Gauss coefficients) in a shifted coordinate system. We shifted the coordinate system northward from Saturn's center of mass along the direction of the spin-axis, in the range of $0.03 R_{S}$ to $0.05 R_{S}$ denoted by $z S$, and solved for the internal Gauss coefficients. We found that no simpler description of the field in terms of Gauss coefficients can be obtained in the 
Z-shifted coordinates, due to the complex nature of Saturn's internal magnetic field in the latitudinal direction. Only the quadrupole moment $g_{2}{ }^{O}$ can be reduced to zero in the $Z$-shifted coordinates with $z S \sim 0.0337 \mathrm{Rs}$; all other even-degree moments (e.g. $g_{4}{ }^{0}, g_{6}{ }^{0}, g_{8}{ }^{0}$ ) remain nonzero (see Fig. S6). In addition, $g_{4}{ }^{0}$ becomes more than an order of magnitude larger in these shifted coordinates compared to that in the Saturn-centered coordinates. The RMS residual remains unchanged as we shift the coordinate system. We therefore do not consider models in shifted coordinates any further.

\section{Implications for Saturn's dynamo and interior}

10 Mathematically, downward continuation of the magnetic field measured outside of a planet towards its interior is strictly valid through regions with no electrical currents $\mathbf{J}$. From the perspective of dynamo action, the downward continuation of the magnetic field is possible through regions with no substantial dynamo action. For Saturn, due to the smooth increase of electrical conductivity as a function of depth $(13,14,59)$, the vigor of dynamo action is expected to rise smoothly with depth (20) and can be quantified by the magnetic Reynolds number, $R m$, which measures the ratio of magnetic field production/modification by flow in an electrically conducting fluid against Ohmic diffusion (60 and Methods). Assuming $1 \mathrm{~cm} \mathrm{~s}^{-1}$ flow and an electrical conductivity model from $(59,61)$, the local $R m$ reaches 10 at $0.77 \mathrm{Rs}, 50$ at $0.725 \mathrm{Rs}$, and 100 at $0.70 \mathrm{R}_{\mathrm{S}}$ (see Fig. S8). For dynamo action in the shallow semi-conducting layer, $R m=20$ is likely to 20 be sufficient given the existence of the main magnetic field originating from the deep dynamo. Thus, the top of the shallow dynamo is likely to fall between 0.77 and 0.725 Rs. We choose 0.75 
Rs as an approximation for the surface of the shallow dynamo; the properties of the downward continued magnetic field are broadly similar in this range of depths.

Figure 7 shows the small-scale $B_{r}$ beyond degree 3 within $+/-60^{\circ}$ latitude at $0.75 R_{S}$ based on the

Cassini 11 model. The small-scale magnetic structures measured along the spacecraft trajectory map to latitudinally banded magnetic structures with amplitude $~ 5000 n T$ and typical spatial scale $\sim 25^{\circ}$ in latitude at $0.75 R S$, which amount to $\sim 5-10 \%$ of the local background field (Fig. S7). The inferred small-scale magnetic field pattern is broadly similar for either a spherical surface with radius $0.75 R_{S}$, or a dynamically flattened elliptical surface with equatorial radius $0.75 R_{S}$ and polar radius $0.6998 R_{S}$ (Figure S9). The magnetic power in the low-degree (degree 1 - 3) part of the ML spectrum still greatly exceeds that in the higher degree part when evaluated at $0.75 R_{S}$ : the dipole power is one order of magnitude larger than the octupole power, while the octupole power is almost two orders of magnitude larger than those of degree $4-11$. The orders-of-magnitude difference between the low-degree power and high-degree power at $0.75 R_{S}$ suggests that the latitudinally banded magnetic perturbations could originate from a secondary dynamo action in the semiconducting region of Saturn (20). This secondary dynamo action likely only produces perturbations on top of the primary deep dynamo, as suggested by a kinematic mean-field dynamo model (20). Fully three-dimensional self-consistent giant planet dynamo models with deep differential rotation (zonal flows) also produced latitudinally banded magnetic fields $(62,63)$.

20 The dynamo $\alpha$-effect, which describes the generation of a poloidal magnetic field from a toroidal magnetic field (see 64 for poloidal and toroidal magnetic field) by rotating convective motion, is likely to be of the traditional mean-field type in the shallow dynamo $(16,17,20)$. The validity of 
the traditional $\alpha$-effect has been extensively discussed in (20). In this scenario, no substantial lowdegree non-axisymmetric magnetic field is expected from the shallow dynamo.

From an empirical perspective, some but not all, of the high-degree magnetic moments could still have a deep interior origin. One extreme interpretation of the Cassini 11 model is to regard the magnetic moments up to degree 9 as entirely being of deep interior origin, because the magnetic power of degrees 7 and 9 is within a factor of three of that of degree 3 when evaluated at $0.50 R_{S}$. When discussing internal magnetic fields well below $0.75 R_{S}$, we have i) treated the shallow dynamo as a small perturbation and ii) implicitly assumed stable stratification between the surface of the deep dynamo and the shallow dynamo. Under this assumption, there exists no local dynamo $\alpha$-effect between the surface of the deep dynamo and the shallow dynamo. Although the $\omega$-effect, which describes the generation of the toroidal magnetic field from differential rotation acting on the poloidal magnetic field, likely operates in the stably stratified layer, the $\omega$-effect will not affect the poloidal component of the magnetic field. Downward continuation of the magnetic field well

15 below $0.75 \mathrm{Rs}_{\mathrm{S}}$ can only be justified if there are no effective electrical currents generating the poloidal magnetic field in the layer between the surface of the deep dynamo and the shallow dynamo.

Figure S10 shows the radial magnetic field as a function of latitude at $0.50 R_{s}$ computed from the

20 Gauss coefficients of the Cassini 11 model up to degree 9. It can be seen that the radial component of Saturn's magnetic field at $0.50 R_{S}$ is strongly concentrated towards high-latitude, leaving minimum magnetic flux at latitudes below \pm 45 degrees, a weaker version of which is evident from 
the magnetic moments up to degree 3 (Figure S10). Figure S10 also suggests the possibility of weak magnetic field at the poles of Saturn near $0.50 R_{S}$. In this alternative interpretation, magnetic moments beyond degree 9 would be of shallower origin. This highlights the ambiguity in the separation of magnetic moments of shallow origin from those of deep origin, which cannot be resolved by MAG data alone.

The main dynamo of Saturn does appear to be deeply buried, i.e. originating from a depth deeper than the expected full metallization of hydrogen at $\sim 0.64 R_{S}(13)$. The surface strength of Saturn's internal magnetic field is more than one order of magnitude weaker than that of Jupiter (65-68). From dynamo scaling analysis, Saturn's magnetic field appears to be weaker than expected from both force-balance considerations and energy-balance considerations if the outer boundary of the deep dynamo is much shallower than $0.40 R_{S}(61)$. The most widely accepted theoretical explanation for Saturn's highly axisymmetric magnetic field $(50,51)$ suggests that differential rotation in a stably stratified and electrically conducting layer above the deep dynamo region electromagnetically filters out non-axisymmetric magnetic moments. Although three-dimensional numerical dynamo simulations $(52,53)$ implementing stable stratification and differential rotation have only produced an average dipole tilt of $0.6^{\circ}$, about two orders of magnitude larger than the newly derived guide value $0.0095^{\circ}$, the magnetic Reynolds number in these $3 \mathrm{D}$ numerical dynamo simulations $(52,53)$ are only in the range of 100 - 500, orders of magnitude smaller than possible

20 values ( 30000) inside the metallic hydrogen layer of Saturn (69). At present, we regard the extreme axisymmetry of Saturn's magnetic field as a continuing enigma of the inner workings of Saturn. 


\section{References and Notes:}

1. D. J. Stevenson, Planetary magnetic fields. Earth and Planetary Science Letters 208, 1-11 (2003).

2. E. J. Smith, L. Davis, D. E. Jones, P. J. Coleman, D. S. Colburn, P. Dyal, C. P. Sonett, Saturn's magnetic field and magnetosphere. Science 207, 407-410 (1980).

3. M. H. Acunã, N. F. Ness., The magnetic field of Saturn - Pioneer 11 observations. Science 207, 444 - 446 (1980).

4. J. E. P. Connerney, N. F. Ness, M. H. Acunã, Zonal harmonic model of Saturn's magnetic field from Voyager 1 and 2 observations. Nature 298, 44 - 46 (1982).

5. J. E. P. Connerney, M. H. Acunã, N. F. Ness, The Z3 model of Saturn's magnetic field and the Pioneer 11 vector helium magnetometer observations. J. Geophys. Res. 89, 7541-7544 (1984).

6. L. Davis, E. J. Smith, New models of Saturn's magnetic field using Pioneer 11 Vector Helium Magnetometer data. J. Geophys. Res. 91, 1373 - 1380 (1986).

7. L. Davis, Jr., E. J. Smith, A model of Saturn's magnetic field based on all available data. J. Geophys. Res. 95, 15257 - 15261 (1990).

8. M. K. Dougherty, S. Kellock, D. J. Southwood, A. Balogh, E. J. Smith, B. T. Tsurutani, B. Gerlach, K.-H. Glassmeier, F. Gleim, C. T. Russell, G. Erdos, F. M. Neubauer, S. W. H. Cowley, The Cassini Magnetic field Investigation. Space Science Reviews 114, 331 - 383 (2004). 
9. M. K. Dougherty, N. Achilleos, N. Andre, C. S. Arridge, A. Balogh, C. Bertucci, M. E. Burton, S. W. H. Cowley, G. Erdos, G. Giampieri, K. -H. Glassmeier, K. K. Khurana, J. Leisner, F. M. Neubauer, C. T. Russell, E. J. Smith, D. J. Southwood, B. T. Tsurutani, Cassini magnetometer observations during Saturn Orbital Insertion. Science 307, 1266 - 1270 (2005).

10. M. E. Burton, M. K. Dougherty, C. T. Russell, Models of Saturn's internal planetary magnetic field based on Cassini observations. Planet. Space Sci. 57, 1706 - 1713 (2009).

11. H. Cao, C. T. Russell, U. R. Christensen, M. K. Dougherty, M. E. Burton, Saturn's very axisymmetric magnetic field: No detectable secular variation or tilt. Earth and Planetary Science Letters 304, 22 - 28 (2011).

12. H. Cao, C. T. Russell, J. Wicht, U. R. Christensen, M. K. Dougherty, Saturn's high degree magnetic moments: Evidence for a unqiue planetary dynamo. Icarus 221, 388 - 394 (2012).

13. S. T. Weir, A. C. Mitchell, W. J. Nellis, Metallization of Fluid Molecular Hydrogen at 140 GPa (1.4 Mbar). Phys. Rev. Lett. 76, 11, 1860 - 1863 (1996).

14. M. Zaghoo, I. F. Silvera, Conductivity and dissociation in liquid metallic hydrogen and implications for planetary interiors, Proceedings of the National Academy of Sciences, 114 (45) 11873-11877, doi: 10.1073/pnas.1707918114 (2018).

15. E. N. Parker, Hydromagnetic dynamo models. Astrophys. J. 122, 293, (1955).

16. F. Krause, K. -H. Rädler, Mean-Field Magnetohydrodynamics and Dynamo Theory. (Pergamon 1980).

17. H. K. Moffatt, Magnetic Field Generation in Electrically Conducting Fluids (Cambridge University Press 1978). 
18. T. G. Cowling, The magnetic field of sunspots. Monthly Notice of Royal Astronomical Society 94, 39 - 48 (1933).

19. P. Charbonneau, Solar and Stellar Dynamos: Saas-Fee Advanced Course 39 Swiss Society for Astrophysics and Astronomy (Springer-Verlag Berlin Heidelberg 2013).

20. H. Cao, D. J. Stevenson, Zonal flow magnetic field interaction in the semi-conducting region of giant planets. Icarus 296, 59 - 72 (2017).

21. D. J. Southwood, M. K. Dougherty, A. Balogh, S. W. H. Cowley, E. J. Smith, B. T Tsurutani, C. T. Russell, G. L. Siscoe, G. Erdos, K. -H. Glassmeier, F. Gleim, F. M. Neubauer, Magnetometer measurements from the Cassini Earth swing-by. J. Geophys. Res. 106, A12, 30109, doi:10.1029/2001JA900110 (2001).

22. E. J. Bunce, S. W. H. Cowley, I. I. Alexeev, C. S. Arridge, M. K. Dougherty, J. D. Nichols, and C. T. Russell, Cassini observations of the variation of Saturn's ring current parameters with system size. J. Geophys. Res. 112, A10202, doi:10.1029/2007JA012275 (2007).

23. J. E. P. Connerney, M. H. Acunã, and N. F. Ness, Saturn's ring current and inner magnetosphere, Nature 292, 724- 726 (1981).

24. J. E. P. Connerney, M. H. Acunã, and N. F. Ness, Currents in Saturn's magnetosphere, J. Geophys. Res. 88, 8779-8789 (1983).

25. G. Giampieri, M. Dougherty, Modeling of the ring current in Saturn's magnetosphere. Annales Geophysicae 22, 653 - 659 (2004).

26. T.M. Edwards, E.J. Bunce, S.W.H. Cowley, A note on the vector potential of Connerney et al.'s model of the equatorial current sheet in Jupiter's magnetosphere. Planet. Space Sci. 49, 1115-1123 (2001). 
27. J. F. Carbary, D. G. Mitchell, Periodicities in Saturn's magnetosphere. Rev. Geophys. 51, 130, doi:10.1002/rog.20006 (2013).

28. D. J. Southwood, S.W.H. Cowley, The origin of Saturn magnetic periodicities: Northern and southern current systems, J. Geophys. Res. 119, 1563-1571, doi: 10.1002/2013JA019632, (2014)

29. D. A. Gurnett, A. Lecacheux, W. S. Kurth, A. M. Persoon, J. B. Groene, L. Lamy, P. Zarka, J. F. Carbary, Discovery of a north-south asymmetry in Saturn's radio rotation period. Geophys. Res. Lett. 36, L16102, doi: 10.1029/2009GL039621 (2009).

30. D. J. Andrews, A. J. Coates, S. W. H. Cowley, M. K. Dougherty, L. Lamy, G. Provan, P. Zarka, Magnetospheric period oscillations at Saturn: Comparison of equatorial and high latitude magnetic field periods with north and south SKR periods. J. Geophys. Res. 115, 1363 A12252, doi: 10.1029/2010JA015666 (2010).

31. D. J. Southwood, Direct evidence of differences in magnetic rotation rate between Saturn's northern and southern polar regions. J. Geophys. Res. 116, 1-11. doi:10.1029/2010JA016070 (2011).

32. G. J. Hunt, G. Provan, S. W. H. Cowley, M. K. Dougherty, D. J. Southwood, Saturn's planetary period oscillations during the closest approach of Cassini's ring-grazing orbits. Geophysical Research Letters 45, 4692-4700. https://doi.org/10.1029/2018GL077925 (2018).

20 33. G. Provan, S. W. H. Cowley, T. J. Bradley, E. J. Bunce, G. J. Hunt, M. K. Dougherty, Planetary period oscillations in Saturn's magnetosphere: Cassini magnetic field observations over the northern summer solstice interval. Journal of Geophysical Research: Space Physics 123, 3859 - 3899. doi:10.1029/2018JA025237 (2018). 
34. P. Galopeau, A. Lecacheux, Variations of Saturn's radio rotation period measured at kilometer wavelengths. J. Geophys. Res. 105, 13089-13101 (2000).

35. D. A. Gurnett, J. B. Groene, A. M. Persoon, J. D. Menietti, S.-Y. Ye, W. S. Kurth, R. J. MacDowell, A. Lecacheux, The reversal of the rotational modulation rates of the north and south components of Saturn kilometric radiation near equinox. Geophys. Res. Lett. 37, L24101, doi:10.1029/2010GL045796 (2010).

36. G. Provan, S. W. H. Cowley, L. Lamy, E. J. Bunce, G. J. Hunt, P. Zarka, M. K. Dougherty, Planetary period oscillations in Saturn's magnetosphere: Coalescence and reversal of northern and southern periods in late northern spring. J. Geophys. Res. 121, 9829-9862, 1500, doi:10.1002/2016JA023056 (2016).

37. X. Jia, M. G. Kivelson, T. I. Gombosi, Driving Saturn's magnetospheric periodicities from the upper atmosphere/ionosphere. J. Geophys. Res. 117, A04215,1460 doi:10.1029/2011JA017367 (2012).

38. X. Jia, M. G. Kivelson, Driving Saturn's magnetospheric periodicities from the upper atmosphere/ionosphere: Magnetotail response to dual sources. J. Geophys. Res. 117, A11219, doi:10.1029/2012JA018183 (2012).

39. J. D. Anderson, G. Schubert, Saturn's Gravitational Field, Internal Rotation, and Interior Structure. Science 317, 5843, 1384 - 1387, doi:10.1126/science.1144835 (2007).

40. P. L. Read, T. E. Dowling, G. Schubert, Saturn's rotation period from its atmospheric planetary-wave configuration. Nature 460, 7225, 608-610, doi:10.1038/nature08194 (2009).

41. R. Helled, E. Galanti, Y. Kaspi, Saturn's fast spin determined from its gravitational field and oblateness. Nature 520, 7546, 202-204, doi:10.1038/nature14278, (2015). 
42. S.W.H. Cowley, E.J. Bunce, J.M. O’Rourke, A simple quantitative model of plasma flows and currents in Saturn's polar ionosphere, J. Geophys. Res. 109, A05212, doi: 10.1029/2003JA010375, (2004).

43. G. J. Hunt, S. W. H. Cowley, G. Provan, E. J. Bunce, I. I. Alexeev, E. S. Belenkaya, V. V. Kalegaev, M. K. Dougherty, A. J. Coates, Field-aligned currents in Saturn's southern nightside magnetosphere: Sub-corotation and planetary period oscillation components. $J$. Geophys. Res. 119, 9847-9899, doi: 10.1002/2014JA020506 (2014).

44. G. J. Hunt, S. W. H. Cowley, G. Provan, E. J. Bunce, I. I. Alexeev, E. S. Belenkaya, V. V. Kalegaev, M. K. Dougherty, A. J. Coates, Field-aligned currents in Saturn's northern nightside magnetosphere: Evidence for inter-hemispheric current flow associated with planetary period oscillations. J. Geophys. Res. 120, 7552-7584, doi: 10.1002/2015JA021454 (2015).

45. B. A. Archinal, C. H. Acton, M. E., A’Hearn, A. Conrad, G. J. Consolmagno, et al., Report of the IAU Working Group on Cartographic Coordinates and Rotational Elements: 2015. Celestial Mechanics and Dynamical Astronomy, 130, 3, 22, doi:10.1007/s10569-017-9805-5 (2018).

46. G. J. Hunt, S. W. H. Cowley, G. Provan, E. J. Bunce, I. I. Alexeev, E. S. Belenkaya, V. V. Kalegaev, M. K. Dougherty, A. J. Coates, Field-aligned currents in Saturn's northern nightside magnetosphere: Evidence for inter-hemispheric current flow associated with planetary period oscillations. J. Geophys. Res. 120, 7552-7584, doi: 10.1002/2015JA021454 (2015).

47. G. J. Hunt, G. Provan, E. J. Bunce, S. W. H. Cowley, M. K. Dougherty, D. J. Southwood, Field-aligned currents in Saturn's magnetosphere: Observations from the F-ring orbits. 
Journal of Geophysical Research: Space Physics 123, 3806-3821, doi: 10.1029/2017JA025067 (2018).

48. K. K. Khurana, M. K. Dougherty, G. Provan, G. J. Hunt, M. G. Kivelson, S. W. H. Cowley, D. J. Southwood, C. T. Russell, Discovery of atmospheric-wind-driven electric currents in Saturn's magnetosphere in the gap between Saturn and its rings, Geophysical Research Letters, doi:10.1029/2018GL078256 (2018).

49. M. E. Burton, M. K. Dougherty, C. T. Russell, Saturn's internal planetary magnetic field. Geophys. Res. Lett., 37, L24105, doi:10.1029/2010GL045148 (2010).

50. D. J. Stevenson, Saturn's luminosity and magnetism. Science 208, 746-748 (1980).

51. D. J. Stevenson, Reducing the non-axisymmetry of a planetary dynamo and an application to Saturn. Geophys. Astrophys. Fluid Dyn. 21, 113 - 127 (1982).

52. U. R. Christensen, J. Wicht, Models of magnetic field generation in partly stable planetary cores: Application to Mercury and Saturn. Icarus 196, 16 - 34 (2008).

53. S. Stanley, A dynamo model for axisymmetrizing Saturn's magnetic field. Geophysical Research Letters 37, L05201 (2010).

54. D. Gubbins, Time Series Analysis and Inverse Theory for Geophysicists (Cambridge University Press, Cambridge, U.K., 2004).

55. R. Holme, J. Bloxham, The magnetic fields of Uranus and Neptune: Methods and models. J. Geophys. Res. 101, 2177 - 2200 (1996).

20 56. P. Mauersberger, Das Mittel der energiedichte des geomagnetischen Hauptfeldes an der erdoberflasähe und seine säkulare Änderung. Gerlands Beitr. Geophys. 65, 207 - 215 (1956). 
57. F. J. Lowes, Mean-square values on sphere of spherical harmonic vector fields. J. Geophys. Res. 71, 2179 - 2179 (1966).

58. F. J. Lowes, Spatial power spectrum of the main geomagnetic field, and extrapolation to the core. Geophys. J. Int. 36, 717 - 730 (1974).

59. J. J. Liu, P. M. Goldreich, D. J. Stevenson, Constraints on deep-seated zonal winds inside Jupiter and Saturn. Icarus 196, 653 - 664 (2008).

60. The local magnetic Reynolds number is defined as $R m=U_{\text {conv }} H_{d} d \lambda$, where $U_{\text {conv }}$ is the typical convective velocity, $\lambda$ is the magnetic diffusivity, defined as the inverse of the product of electrical conductivity and magnetic permeability, and $H_{\sigma}$ is the conductivity scale-height defined as $H_{\sigma}=\sigma / d \sigma / d r \mid$.

61. U. R. Christensen, Dynamo Scaling Laws and Application to the Planets. Space Science Reviews 152, 565 - 590 (2010).

62. T. Gastine, J. Wicht, L. D. V. Duarte, M. Heimpel, A. Becker, Explaining Jupiter's magnetic field and equatorial jet dynamics, Geophys. Res. Lett. 41, doi:10.1002/2014GL060814 (2014).

63. G. A. Glatzmaier, Computer simulations of Jupiter's deep internal dynamics help interpret what Juno sees, Proceedings of the National Academy of Sciences, 201709125; DOI:10.1073/pnas.1709125115 (2018).

64. Due the divergence free nature of magnetic field, a poloidal-toroidal decomposition of the magnetic field, $\mathbf{B}=\nabla \times\left(\nabla \times \mathrm{P} \mathbf{e}_{\mathbf{r}}\right)+\nabla \times \mathrm{T} \mathbf{e}_{\mathbf{r}}$, is commonly adopted in the dynamo region. The toroidal magnetic field, $\mathbf{B}_{\mathbf{T}}=\nabla \times \mathrm{Te}_{\mathbf{r}}$ has no radial component, and is associated with local 
electrical currents. The poloidal magnetic field $\mathrm{BP}=\nabla \times\left(\nabla \times \mathrm{Pe}_{\mathbf{r}}\right)$ has no azimuthal component if it is axisymmetric.

65. J.E.P. Connerney, M.H. Acuna, N.F. Ness, T. Satoh, New models of Jupiter's magnetic field constrained by the Io flux tube footprint. J. Geophys. Res. 103, 11929 - 11940 (1998).

66. Z. J. Yu, H. K. Leinweber, C. T. Russell, Galileo constraints on the secular variation of the Jovian magnetic field. J. Geophys. Res. 115, E03002 (2010).

67. V. A. Ridley, R. Holme, Modeling the Jovian magnetic field and its secular variation using all available magnetic field observations. J. Geophys. Res. 121, 3, 309 - 337 (2016).

68. K. M. Moore, J. Bloxham, J. E. P. Connerney, J. L. Jørgensen, J. M. G. Merayo, The analysis of initial Juno magnetometer data using a sparse magnetic field representation. Geophys. Res. Lett. 44, doi: 10.1002/2017GL073133 (2017).

69. Assuming $1 \mathrm{~cm} \mathrm{~s}^{-1}$ flow and an electrical conductivity of $2 \times 10^{5} \mathrm{~S} / \mathrm{m}, R m$ associated with a layer with a thickness of $0.2 R s$ would be $\sim 30000$.

\section{Methods}

Un-regularized and regularized inversion of Saturn's internal magnetic field:

The internal magnetic field of Saturn outside the dynamo region is described using Gauss coefficients $\left(g_{n}{ }^{m}, h_{n}{ }^{m}\right)$ where $n$ and $m$ are the spherical harmonic degree and order respectively:

$V^{\mathrm{Int}}=\sum_{n=1} \sum_{m=0}^{l} R_{p}\left(\frac{R_{p}}{r}\right)^{n+1}\left[g_{n}^{m} \cos (m \varphi)+h_{n}^{m} \sin (m \varphi)\right] P_{n}^{m}(\cos \theta)$, 
$\left(B_{r}^{\mathrm{Int}}, B_{\theta}^{\mathrm{Int}}, B_{\varphi}^{\mathrm{Int}}\right)=-\nabla V^{\mathrm{Int}}$

$B_{r}^{\text {Int }}=\sum_{n=1} \sum_{m=0}^{l}(n+1)\left(\frac{R_{p}}{r}\right)^{n+2}\left[g_{n}^{m} \cos (m \varphi)+h_{n}^{m} \sin (m \varphi)\right] P_{n}^{m}(\cos \theta)$,

$B_{\theta}^{\mathrm{Int}}=-\sum_{n=1} \sum_{m=0}^{l}\left(\frac{R_{p}}{r}\right)^{n+2}\left[g_{n}^{m} \cos (m \varphi)+h_{n}^{m} \sin (m \varphi)\right] \frac{d P_{n}^{m}(\cos \theta)}{d \theta}$,

$B_{\varphi}^{\mathrm{Int}}=\sum_{n=1} \sum_{m=1}^{l}\left(\frac{R_{p}}{r}\right)^{n+2} \frac{m}{\sin \theta}\left[g_{n}^{m} \sin (m \varphi)-h_{n}^{m} \cos (m \varphi)\right] P_{n}^{m}(\cos \theta)$.

It can be seen from Eq. 5 that the azimuthal magnetic field of internal origin, $B_{\varphi}{ }^{\text {Int }}$, must be part of a non-axisymmetric $(m \neq 0)$ field pattern.

The forward model can be represented as

data $=$ G model.

For this particular problem, data represents the magnetic field measurements $\left(B_{r}, B_{\theta}, B_{\varphi}\right)$, model represents the Gauss coefficients $\left(g_{n}{ }^{m}, h_{n}{ }^{m}\right)$, and $G$ represents the matrix expression of Eqs. 3 to

5.

In un-regularized inversion, one seeks to minimize the difference between the data and the model field only

$|d a t a-G \operatorname{model}|^{2}$,

15 whereas in regularized inversion, additional constraints are placed on the model parameters. Instead, one seeks to minimize

$\mid$ data $-G$ model $\left.\right|^{2}+\gamma^{2} \mid L$ model $\left.\right|^{2}$, 
in which $L$ represents a particular form of regularization and $\gamma$ is a tunable damping parameter adjusting the relative importance of model constraints.

We seek to minimize the power in the surface integral of the radial component of the magnetic field beyond spherical harmonic degree 3

$5 \quad \int_{r=r_{r e f}} B_{r}^{2} d \Omega=\sum_{n=4} \frac{(n+1)^{2}}{2 n+1}\left(\frac{R_{p}}{r_{r e f}}\right)^{2 n+4}\left[\left(g_{n}^{m}\right)^{2}+\left(h_{n}^{m}\right)^{2}\right]$

at a reference radius $r_{\text {ref. }}$. Thus,

$L=\frac{n+1}{\sqrt{2 n+1}}\left(\frac{R_{p}}{r_{r e f}}\right)^{n+2}$

for $n>3$ and $L=0$ for $n \leq 3$.

After experimenting with different $r_{r e f}$ and $\gamma$, we choose $r_{r e f}=1.0$ and $\gamma=0.6$ which yield smooth magnetic field perturbations when viewed between $1.0 R_{S}$ and $0.75 R_{S}$.

In this framework, the model solution can be computed via

model $=\left(G^{T} G+\gamma^{2} L^{T} L\right)^{-1} G^{T}$ data $=G^{N}$ data,

in which superscript $T$ represents matrix transpose.

The model covariance matrix can be computed from

$\bmod _{c o v}=G^{N}$ data $_{\text {cov }}\left(G^{N}\right)^{T}$

where $d a t a_{c o v}$ is the data covariance matrix, taken to be $\sigma_{\text {data }}{ }^{2} \boldsymbol{I}$, in which $\sigma_{\text {data }}$ is the RMS of the data model misfit and $\boldsymbol{I}$ is the identity matrix (54). 
Electromagnetic induction inside Saturn:

Due to the rapidly radial varying nature of electrical conductivity inside Saturn, the three factors controlling the electromagnetic (EM) induction at Saturn are: the frequency of the inducing field $\omega_{\text {ind }}$, the electrical conductivity $\sigma(r)$ and the electrical conductivity scaleheight $H_{\sigma}=\sigma|d \sigma / d r|$. The EM induction inside Saturn would occur at a depth where the frequency dependent local EM skin depth $d(r, \omega)=\sqrt{2 / \omega_{\text {ind }} \mu_{0} \sigma(r)}$ becomes similar to or less than the local electrical conductivity scaleheight $H_{\sigma}(r)$, where $\mu_{0}$ is the magnetic permeability.

Fig. S4 shows the frequency dependent EM skin depth compared to the electrical conductivity scaleheight inside Saturn, adopting the electrical conductivity profile (51). Two frequencies have been chosen, the rotational frequency of Saturn $\omega_{r}=\frac{2 \pi}{10.5 \text { hours }} \sim 1.7 \times 10^{-4} \mathrm{rad}^{-1}$ and the orbital period of Cassini Grand Finale orbits $\omega_{o}=\frac{2 \pi}{7 \text { Earth days }} \sim 1.0 \times 10^{-5} \operatorname{rad~}^{-1}$. It can be seen from Fig. S4 that EM induction inside Saturn would occur at depth $0.87 R_{S}$ for $\omega_{i n d}$ equal to the rotational frequency of Saturn and at depth $0.86 R_{S}$ for $\omega_{i n d}$ equal to the orbital frequency of Cassini Grand Finale orbits.

15 Thus, the induction depth inside Saturn would likely be $0.87 R_{S}$ or less. A $10 n T$ inducting $B_{Z}$ field would create a time-varying internal dipole field with equatorial strength of $5 n T$ and polar strength of $10 n T$ when evaluated at this depth, which corresponds to a time varying dipole coefficient $g_{1}{ }^{0}$ of $3.3 n T$ when defined with respect to the equatorial radius of Saturn's 1 bar surface. 
Acknowledgments: All authors acknowledge support from the Cassini Project.

\section{Funding:}

Work at Imperial College London was funded by STFC consolidated grant ST/N000692/1. M.K.

D. is funded by Royal Society Research Professorship RP140004. H.C. is funded by the NASA's CDAPS program NNX15AL11G and NASA JPL contract 1579625 . Work at the University of Leicester was supported by STFC grant ST/N000749/1. E.J.B is supported by a Royal Society Wolfson Research Merit Award. Work at UCLA is funded by NASA Jet Propulsion Laboratory contract 1409809. K.K.K is funded by NASA JPL contract 1409806:033. M.G.K is funded by

10 JPL under contract 1416974 at the University of Michigan. M.E.B. and T.A.B. is supported by the Cassini Project.

\section{Author contributions:}

M.K.D. led the instrument team and supervised the data analysis. H.C., K.K.K., S.K. carried out the magnetometer calibration analysis. T.A.B. carried out the spacecraft attitude reconstruction. H.C., G.J.H, G.P. carried out the magnetic field data analysis. M.E.B., E.J.B., S.W.H.C., M.G.K., C.T.R., D.J.S. provided theoretical support and advised on the data analysis. All authors contributed to the writing of the manuscript.

\section{Competing interests:}

No competing interests.

\section{Data and materials availability:}

The derived model parameters are given in Tables 1, S1 and S2. As agreed with NASA, fully calibrated Cassini magnetometer data are released on schedule via the NASA Planetary Data System at https://pds.nasa.gov/. 


\section{List of Supplementary Materials:}

Figures S1-S10

Tables S1-S2 

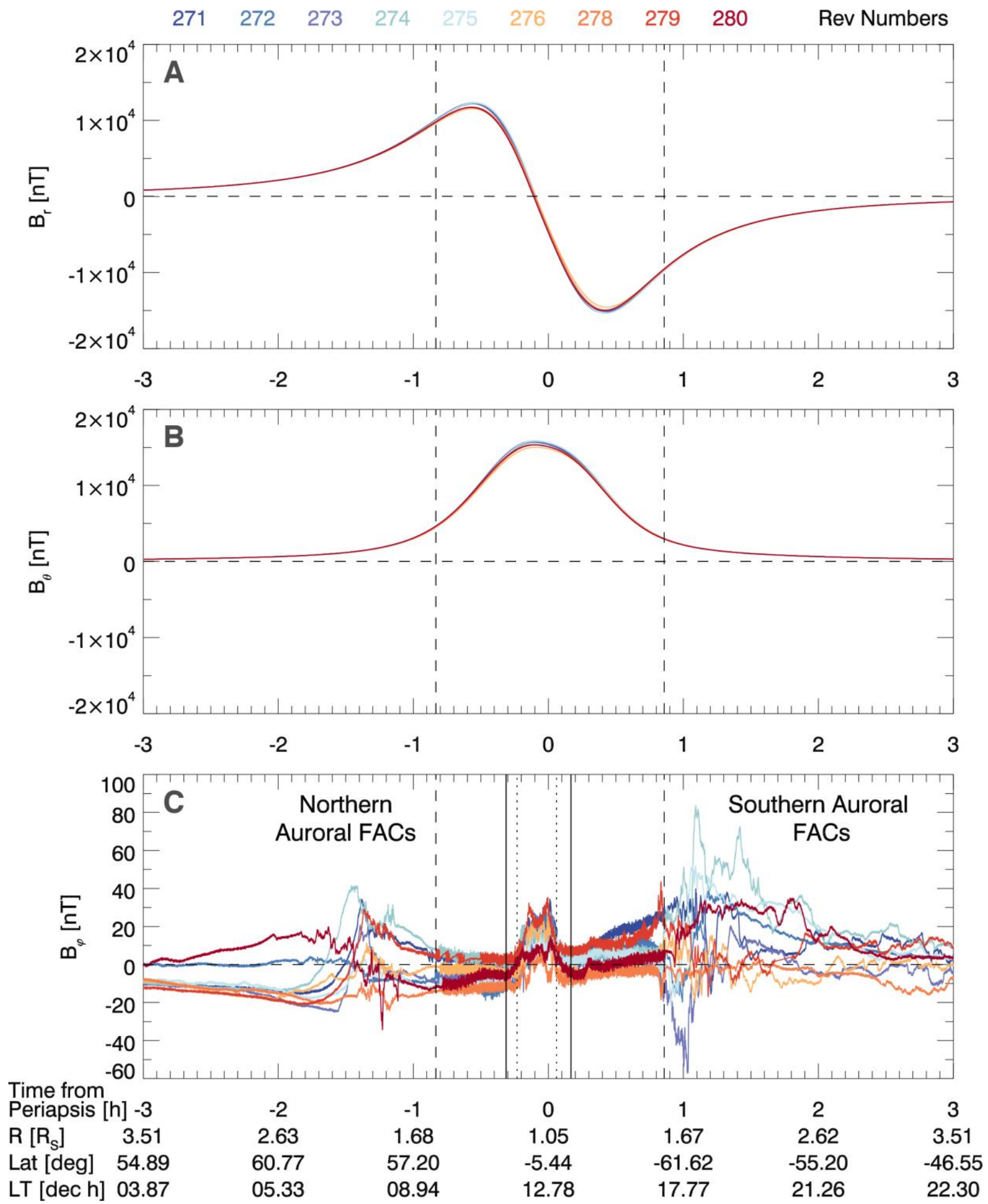

Fig. 1. Vector magnetic field measurements from nine Cassini Grand Finale orbits. The

IAU System III Saturn-centered spherical polar co-ordinates are adopted here. The peak 
measured magnetic field strength is $\sim 18,000 \mathrm{nT}$, while the azimuthal component of the field (plotted on a different scale) is $\sim 1 / 1000$ of the total field outside the high latitude auroral fieldaligned currents (FACs) region which are labelled. Vertical dashed lines indicate the MAG range $3(|\mathrm{~B}|>10,000 \mathrm{nT})$ time period. In the bottom panel the vertical dotted lines mark the inner edge

5 of the D ring, and the solid vertical solid lines mark the outer edge of the D ring mapped magnetically, as described in the text. 


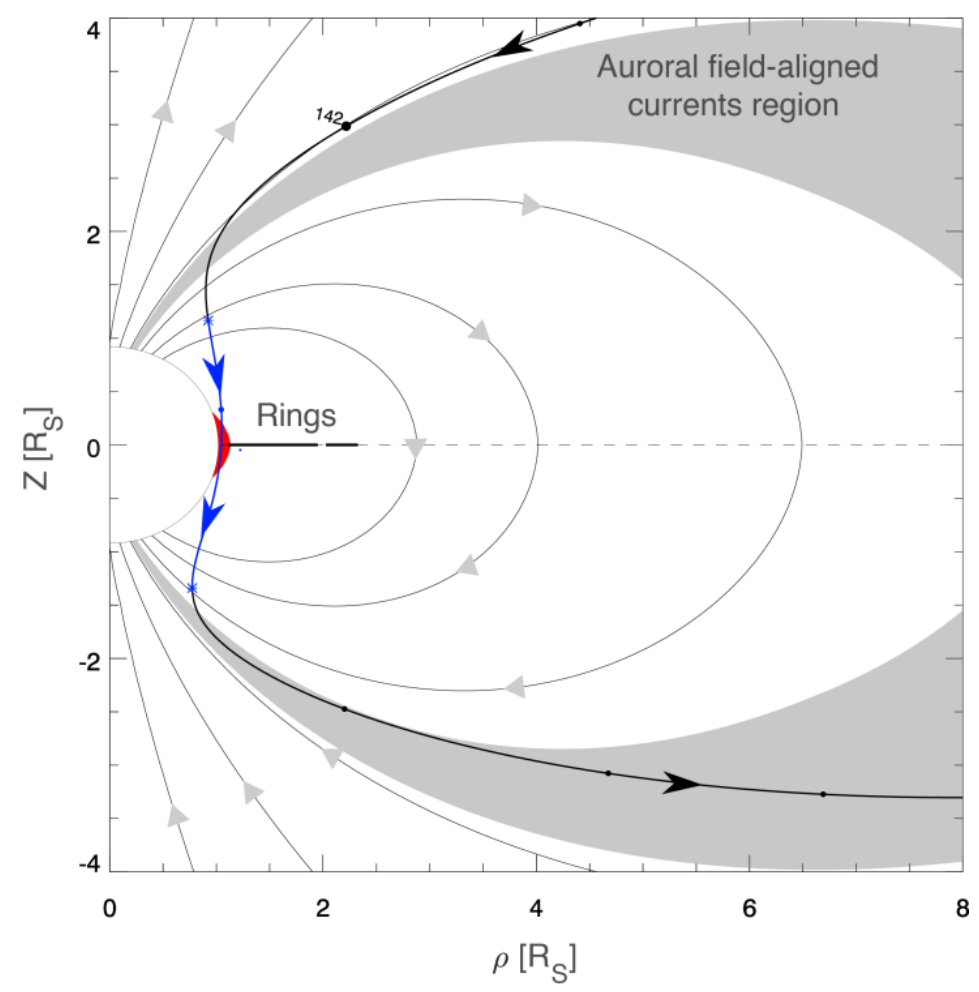

Fig. 2. Cassini's Grand Finale trajectory shown in the meridional plane. The trajectory of Cassini Grand Finale orbit Rev 275 (black/blue trace with arrows) in the $\rho$ - Z cylindrical coordinate system, where $\rho$ is the perpendicular distance from the spin axis and $\mathrm{Z}$ is distance from Saturn's planetary equator, is overlain on the average positions of Saturn's auroral FACs region (gray region) and the newly discovered low-latitude FACs (red region). Thin grey traces with arrows are magnetic field lines. The interval along the trajectory during which the measured magnetic field was above 10,000 nT is highlighted in blue. Small circles are at 3-hour intervals and the beginning of day 142 of year 2017 is shown. 

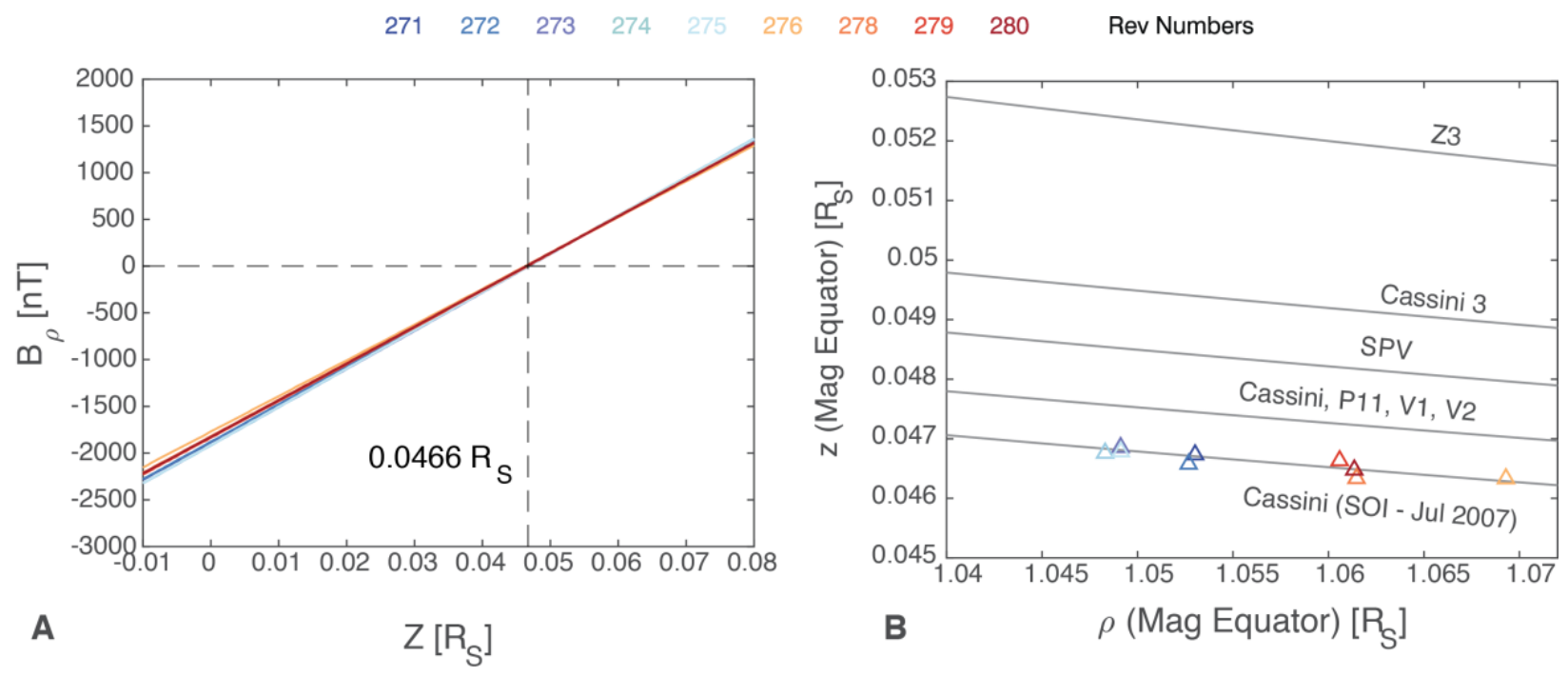

Fig. 3. Saturn's magnetic equator position as directly measured along the Cassini Grand Finale orbits. Panel A shows the measured cylindrical radial component of the magnetic field versus distance from Saturn's planetary equator from nine Cassini Grand Finale orbits. Panel B shows the distribution of the magnetic equator northward displacements (triangles) vs. cylindrical radial distance compared to predictions from existing degree-3 internal field models (lines) $(4,7,10,11)$. The Z3 model is from (4), the Cassini 3 model is from (11), the SPV model is from (7), the Cassini (SOI - Jun 2007) model and the Cassini, P11, V1, V2 model are both from (10). The Cassini (SOI - Jun 2007) model were derived from Cassini SOI data to July 2007 data and the Cassini, P11, V1, V2 model is derived from Cassini SOI to July 2007 data combined with Pioneer 11, Voyager $1 \& 2$ data (10). 

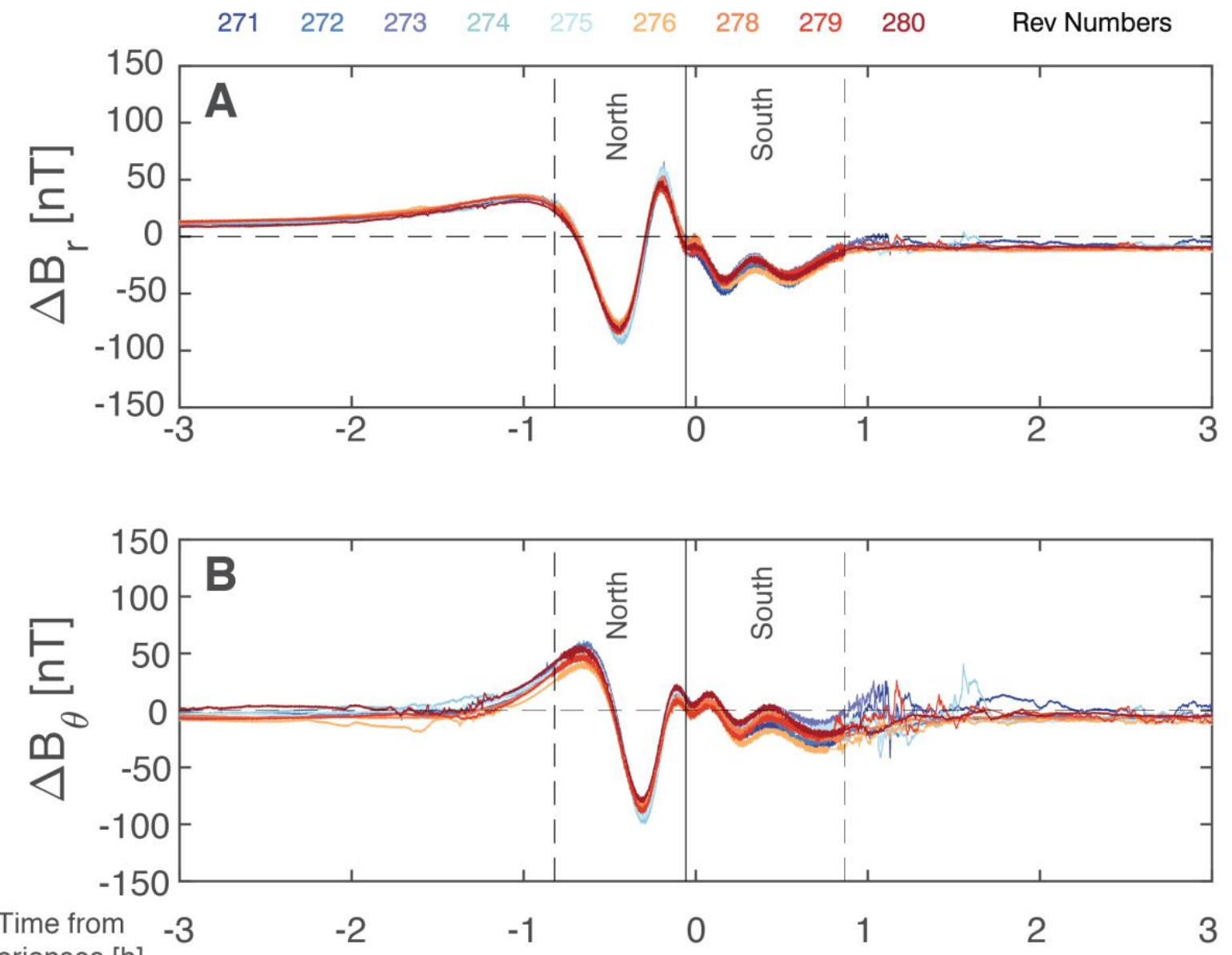

Periapses [h]

$\begin{array}{cccccccc}\mathrm{R}\left[\mathrm{R}_{\mathrm{S}}\right] & 3.51 & 2.63 & 1.68 & 1.05 & 1.67 & 2.62 & 3.51 \\ \text { Lat }[\mathrm{deg}] & 54.89 & 60.77 & 57.20 & -5.44 & -61.62 & -55.20 & -46.55 \\ \text { LT [dec h] } & 03.87 & 05.33 & 08.94 & 12.78 & 17.77 & 21.26 & 22.30\end{array}$

Fig. 4. Saturn's magnetic field beyond spherical harmonic degree 3 as directly measured during the Grand Finale orbits. Shown here are residuals of the radial and meridional magnetic fields from the un-regularized degree 3 internal field model (see Table S1 for the parameters). It can be seen that the residuals are on the order of $100 \mathrm{nT}$, which is 10 times larger than the magnetodisk field. 

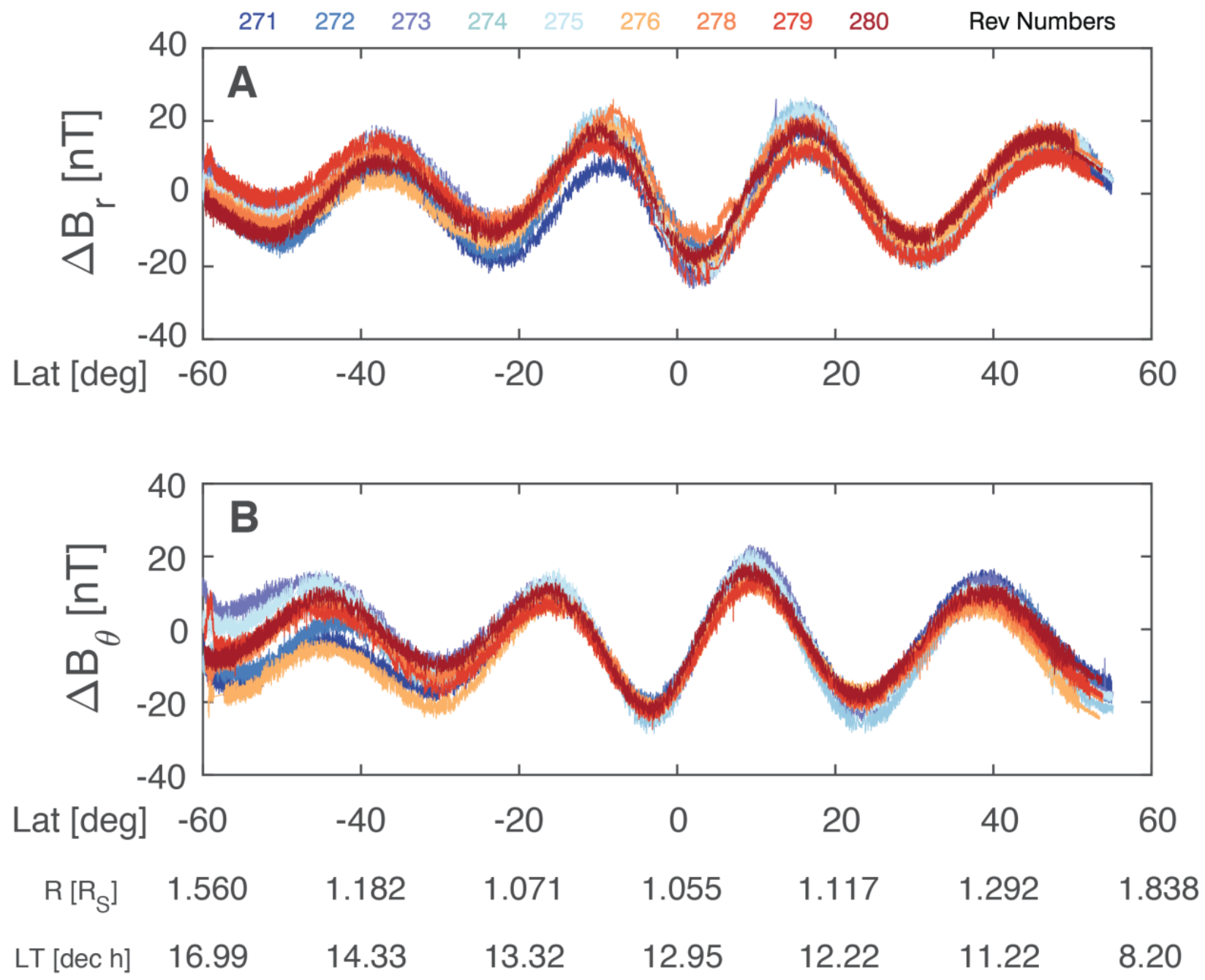

Fig. 5. Saturn's magnetic field beyond spherical harmonic degree 6 as directly measured during the Grand Finale. Shown here are residuals of the radial and meridional magnetic fields from the un-regularized degree 6 internal field model (see Table S1 for the parameters). The field corresponding to the magnetodisk current has also been removed (see Table S2 for the parameters of the magnetodisk current model). Latitudinally banded magnetic structures on the order of $25 n T$ are evident. Orbit to orbit deviations in $B_{\theta}$ between $-60^{\circ}$ and $-40^{\circ}$ latitudes are mostly due to the influences of southern hemisphere high-latitude field aligned currents. 


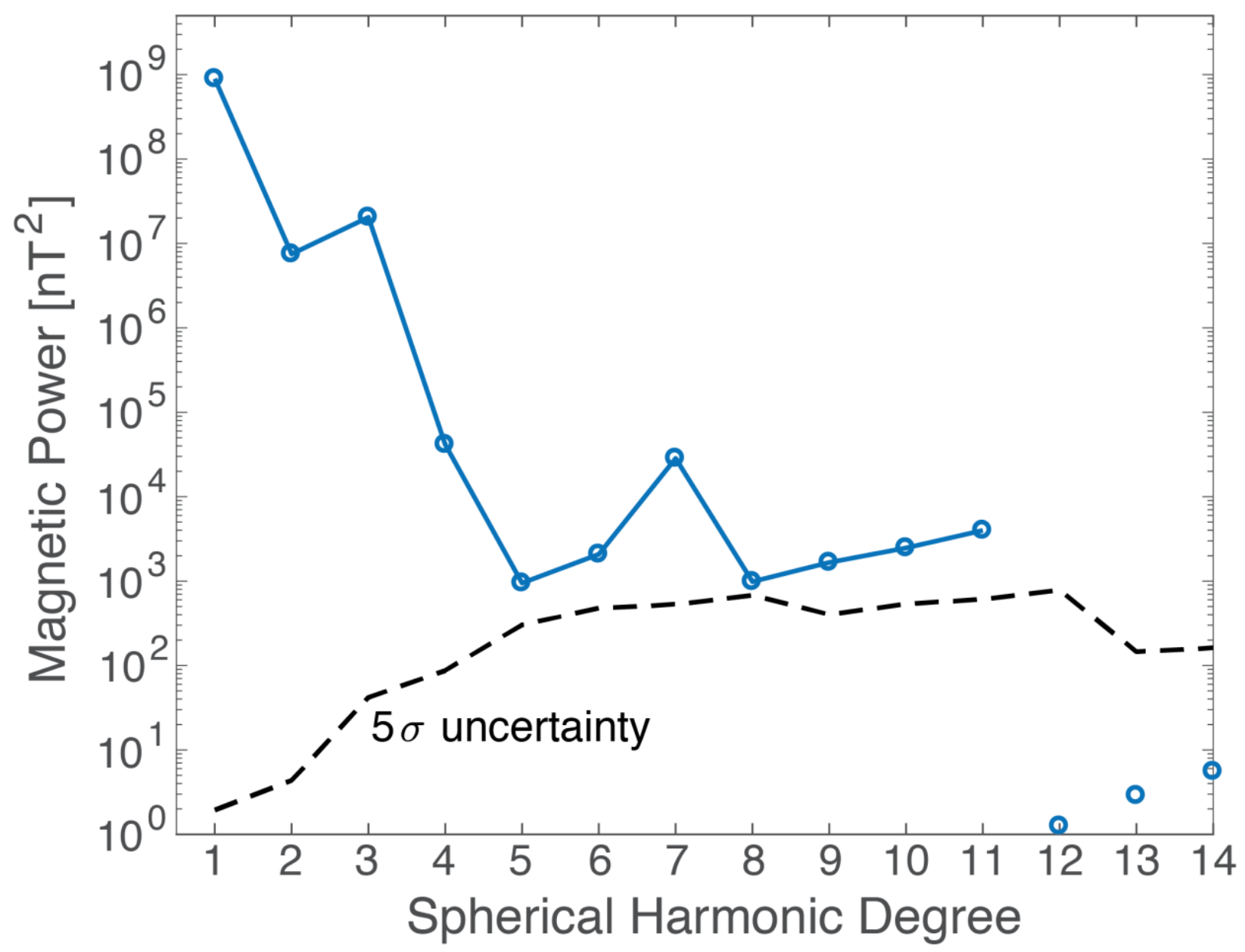

Fig. 6. Magnetic power spectrum of the Cassini 11 model. This new internal field model for Saturn is constructed from nine Cassini Grand Finale orbits with regularized inversion. Central values and five times the formal uncertainties derived from the regularized inversion are shown (see Methods for details). It can be seen that the high-degree moments between degree 4 and degree 11 are on the order of $10-100 n T$, while those above degree 11 are less than $10 n T$, below the derived uncertainty. 


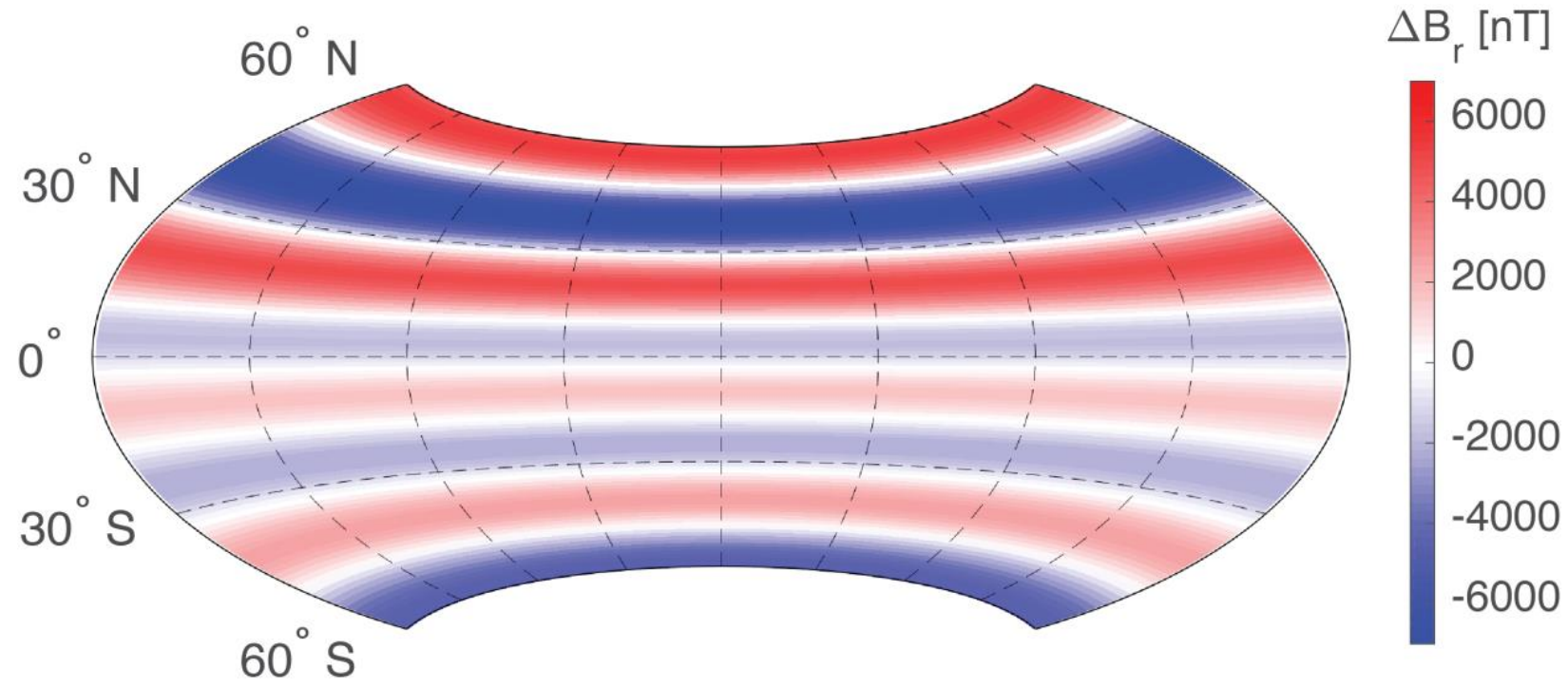

Fig. 7. Small-scale axisymmetric magnetic field of Saturn at $0.75 R_{S}$. The values of $\Delta \mathrm{B}_{\mathrm{r}}$ are computed using the central values of the degree 4 to 11 Gauss coefficients (see Table 1). These small-scale magnetic perturbations at $0.75 R_{S}$ are on the order of $5000 n T$, which amount to $\sim 5$ $10 \%$ of the local background field (see Fig. S6). The plotted latitudinal range corresponds to the region of Cassini MAG range 3 measurements along the Grand Finale orbits.

Table 1. Gauss Coefficients of a new model for Saturn's internal magnetic field, which we refer to as the Cassini 11 model, constructed from nine orbits of Cassini Grand Finale MAG data with 
regularized inversion. The reported uncertainty is five times the formal uncertainties associated with the chosen regularization (see Methods).

\begin{tabular}{|l|l|c|}
\hline Gauss & Value $[n T]$ & Uncertainty $[n T]$ \\
\hline $\mathrm{g}_{1}{ }^{0}$ & & \\
\hline $\mathrm{g}_{2}{ }^{0}$ & 21140.2 & 1.0 \\
\hline $\mathrm{g}_{3}{ }^{0}$ & 1581.1 & 1.2 \\
\hline $\mathrm{g}_{4}{ }^{0}$ & 2260.1 & 3.2 \\
\hline $\mathrm{g}_{5}{ }^{0}$ & 91.1 & 4.2 \\
\hline $\mathrm{g}_{6}{ }^{0}$ & 12.6 & 7.1 \\
\hline $\mathrm{g}_{7}{ }^{0}$ & 17.2 & \\
\hline $\mathrm{g}_{10}{ }^{0}$ & & \\
\hline $\mathrm{g}_{8}{ }^{0}$ & -59.6 & 8.2 \\
\hline $\mathrm{g}_{9}{ }^{0}$ & & \\
\hline & -10.5 & 8.1 \\
\hline & & \\
\hline & & \\
\hline & & \\
\hline & & \\
\hline & & \\
\hline & & \\
\hline
\end{tabular}




\begin{tabular}{|l|l|c|}
\hline $\mathrm{g}_{12}{ }^{0}$ & 0.3 & 7.7 \\
\hline RMS Residual & 6.2 & \\
\hline
\end{tabular}




\section{Science $\triangle 1$ AAAS}

5

\section{Supplementary Materials for}

Saturn's magnetic field revealed by the Cassini Grand Finale Michele K. Dougherty ${ }^{1}$, Hao Cao ${ }^{2,3,1}$, Krishan K. Khurana ${ }^{4}$, Gregory J. Hunt ${ }^{1}$, Gabrielle Provan $^{5}$, Stephen Kellock ${ }^{1}$, Marcia E. Burton ${ }^{6}$, Thomas A. Burk ${ }^{6}$, Emma J. Bunce ${ }^{5}$, Stanley W. H. Cowley ${ }^{5}$, Margaret G. Kivelson ${ }^{4,7}$, Christopher T. Russell ${ }^{4}$ and David J. Southwood ${ }^{1}$.

10

${ }^{1}$ Physics Department, The Blackett Laboratory, Imperial College London, SW7 2AZ, UK.

${ }^{2}$ Department of Earth and Planetary Sciences, Harvard University, 20 Oxford Street, Cambridge, MA 02138, USA.

${ }^{3}$ Division of Geological and Planetary Sciences, California Institute of Technology, $1200 \mathrm{E}$ 15 California Blvd, Pasadena, CA 91125, USA.

${ }^{4}$ Department of Earth, Planetary, and Space Sciences, University of California, Los Angeles, 90025, USA.

${ }^{5}$ Department of Physics and Astronomy, University of Leicester, Leicester, LE1 7RH, UK.

${ }^{6}$ Jet Propulsion Laboratory, California Institute of Technology, 4800 Oak Grove Dr, Pasadena, 20 CA 91109, USA.

${ }^{7}$ Department of Climate and Space Sciences and Engineering, University of Michigan, Ann Arbor, MI 40109, USA.

Correspondence to: m.dougherty@imperial.ac.uk

Figs. S1 to S10

Tables $\mathrm{S} 1$ to $\mathrm{S} 2$ 


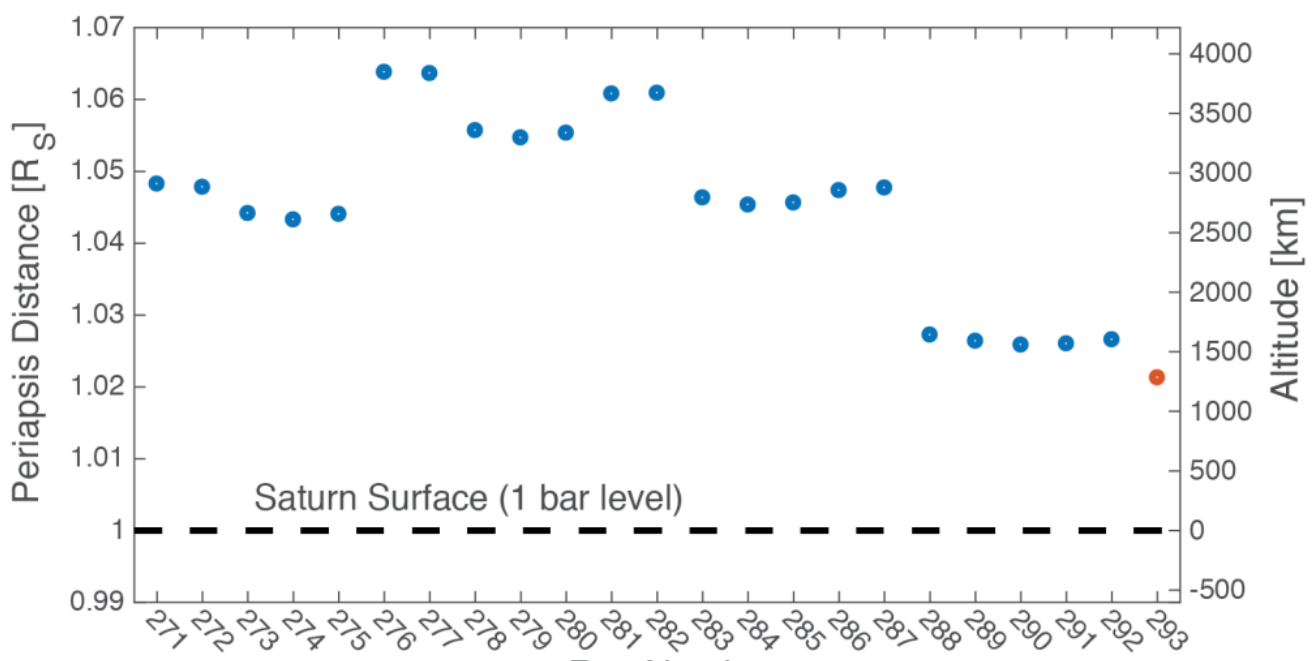

A

Rev Number
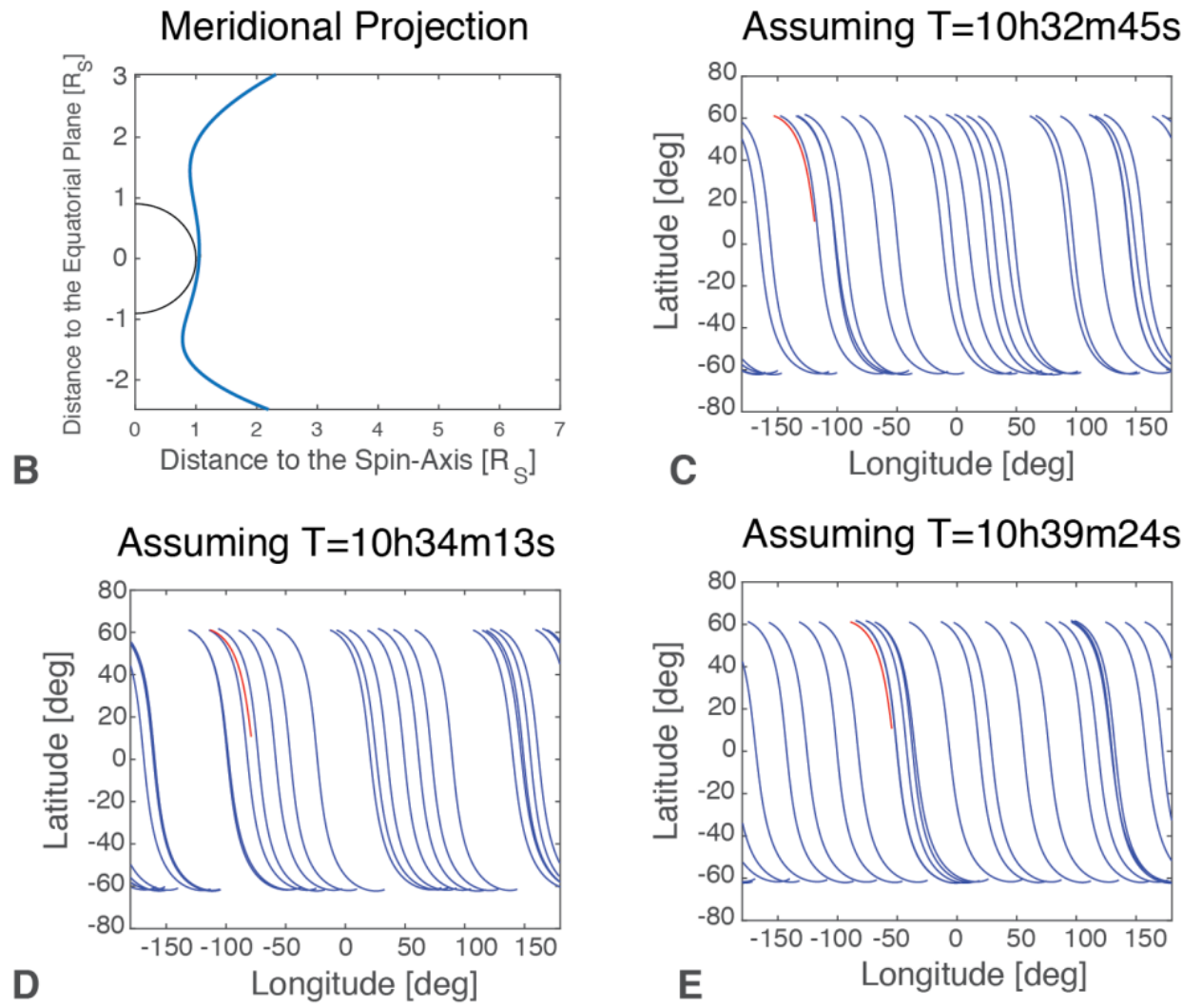

Fig. S1. Characteristics of the trajectory of Cassini Grand Finale orbits. Panel A shows the periapse distance for each orbit. Panel B shows the meridional projection of the first Grand Finale orbit (Rev 271). Panel C-E show and latitude-longitude coverage of the trajectory of the Cassini Grand Finale orbits for three different assumed rotation periods. 

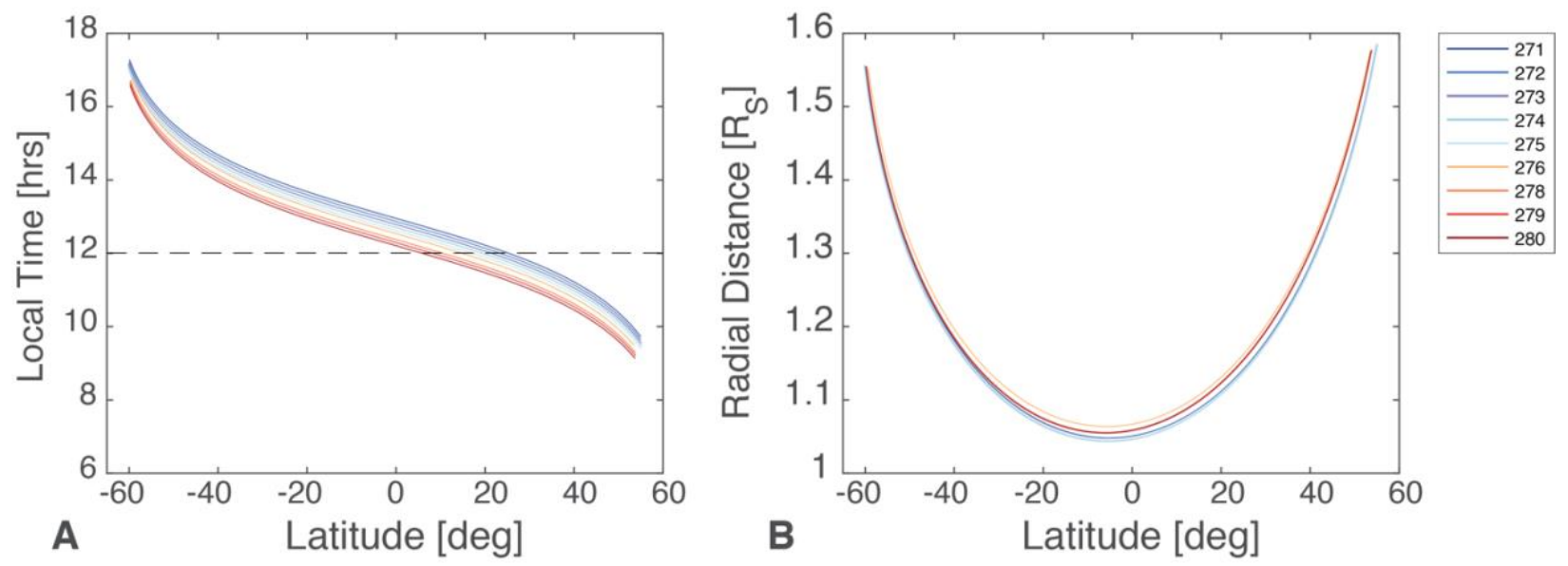

Fig. S2. Characteristics of the trajectory of nine Cassini Grand Finale orbits. Panel A shows local time versus latitude, while panel B shows radial distance versus latitude. The same colour code applies to both panels. We only show the portion of the trajectory when the Cassini magnetometer was in range 3 (magnetic field strength $>10,000 n T$ ). The spacecraft was in the morning (afternoon) sector in the northern (southern) hemisphere. 


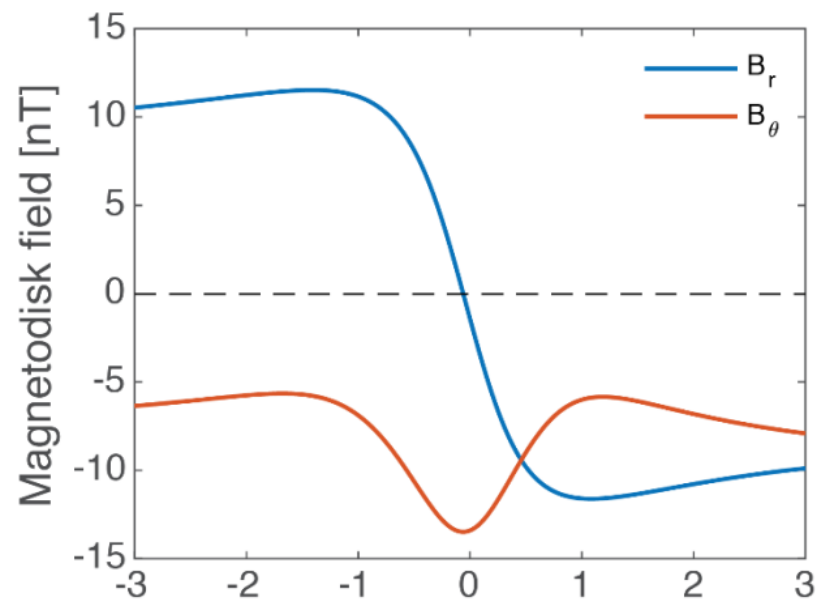

A

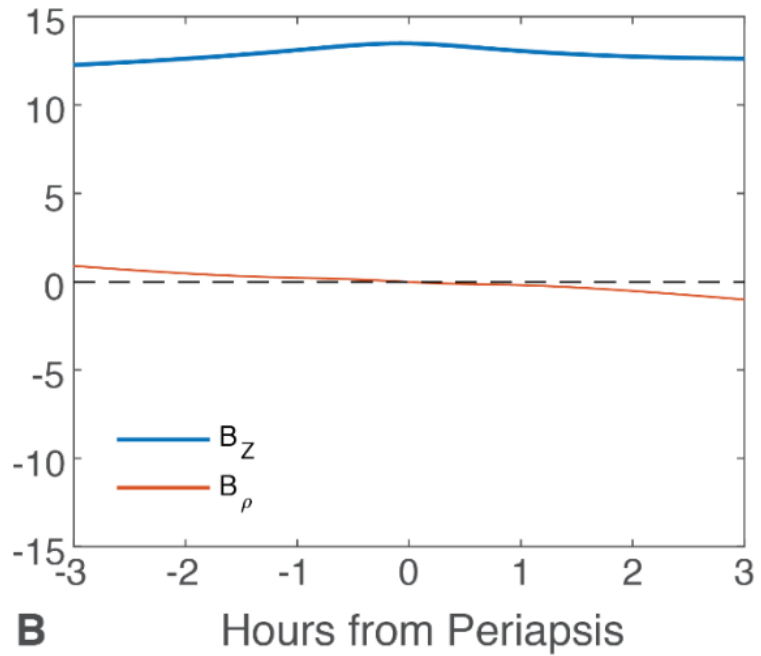

B Hours from Periapsis

Fig. S3. The expected magnetodisk field along the Cassini Grand Finale orbits. The same field is shown in two different coordinate systems: Saturn-centered spherical coordinates (Panel A) and cylindrical coordinates (Panel B). The trajectory of Cassini Rev 271 was used, and the following parameters for the magnetodisk field model were adopted $\left(\boldsymbol{a}, \boldsymbol{b}, \boldsymbol{D}, \boldsymbol{\mu}_{\mathbf{0}} I\right)=$ (6. $\left.5 R_{S}, 25 R_{S}, 2.5 R_{S}, 48.5 n T\right)$. See references (22-26) for definition of the parameters of the magnetodisk field. It can be seen from the right panel that the magnetodisk field along the \pm 3 hours from periapsis of the Cassini Grand Finale trajectory is close to that of a uniform field in the direction parallel to the spin-axis, $\boldsymbol{B}_{\boldsymbol{Z}}$, around $12 \mathrm{nT}$. The expected magnetodisk field is very small in the cylindrical radial direction: less than $0.25 \mathrm{nT} \boldsymbol{B}_{\rho}$ within $+/-1$ hour from the periapsis. This makes $\boldsymbol{B}_{\boldsymbol{\rho}}$ the cleanest component to probe the internal magnetic field of Saturn. 


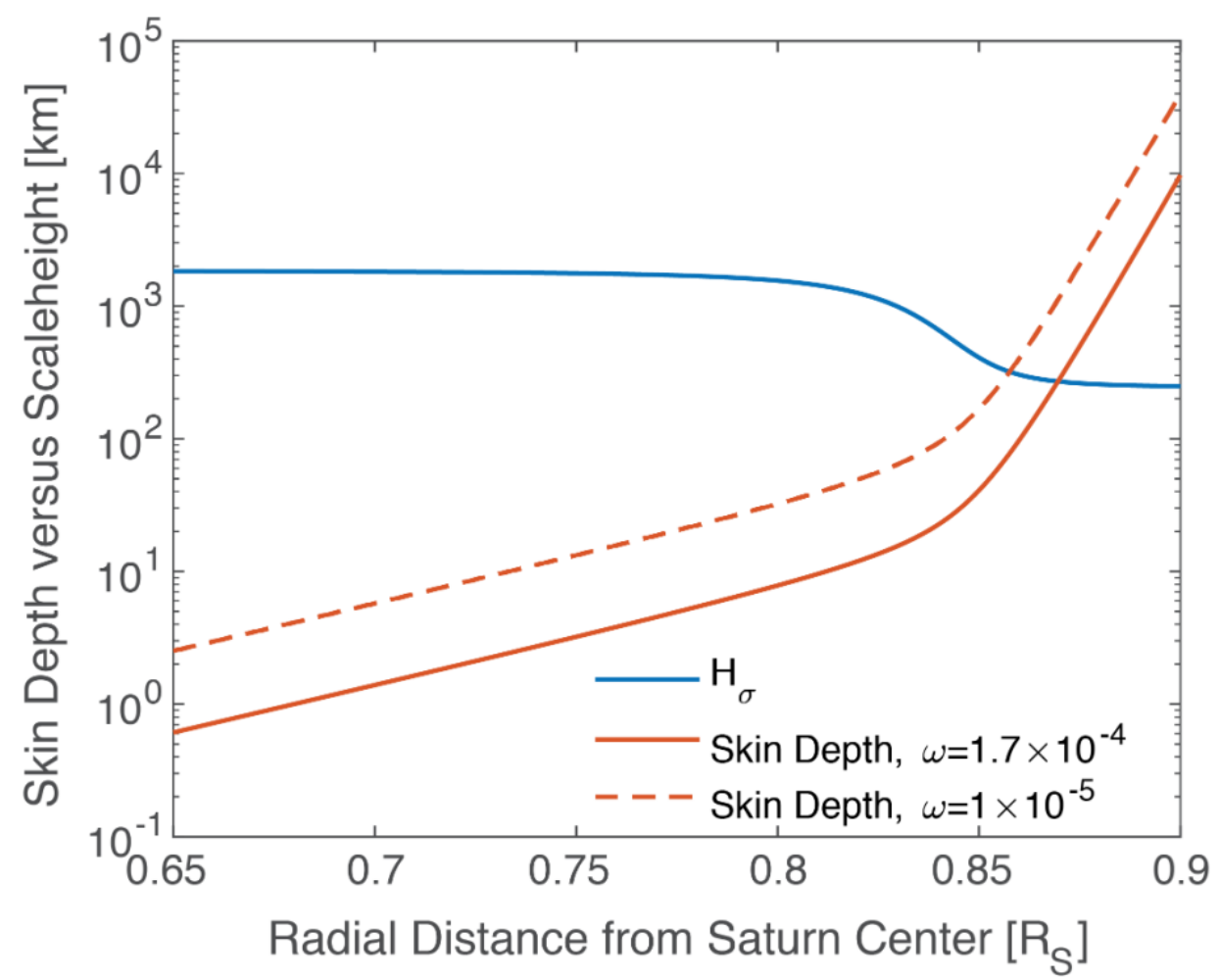

Fig. S4. The frequency dependent electromagnetic (EM) skin depth compared to the electrical conductivity scaleheight inside Saturn. The electrical conductivity profile of (59) is adopted for the calculations here. The two frequencies shown here correspond to the rotational frequency of Saturn $\omega_{r}=\frac{2 \pi}{10.5 \text { hours }} \sim 1.7 \times 10^{-4}$ and the orbital period of Cassini Grand Finale orbits $\omega_{o}=\frac{2 \pi}{7 \text { Earth days }} \sim 1.0 \times 10^{-5}$. It can be seen that EM induction inside Saturn would occur at depth $0.87 R_{S}$ for $\omega_{\text {ind }}$ equal to the rotational frequency of Saturn and at depth $0.86 R_{S}$ for $\omega_{\text {ind }}$ equal to the orbital period of Cassini Grand Finale orbits. 


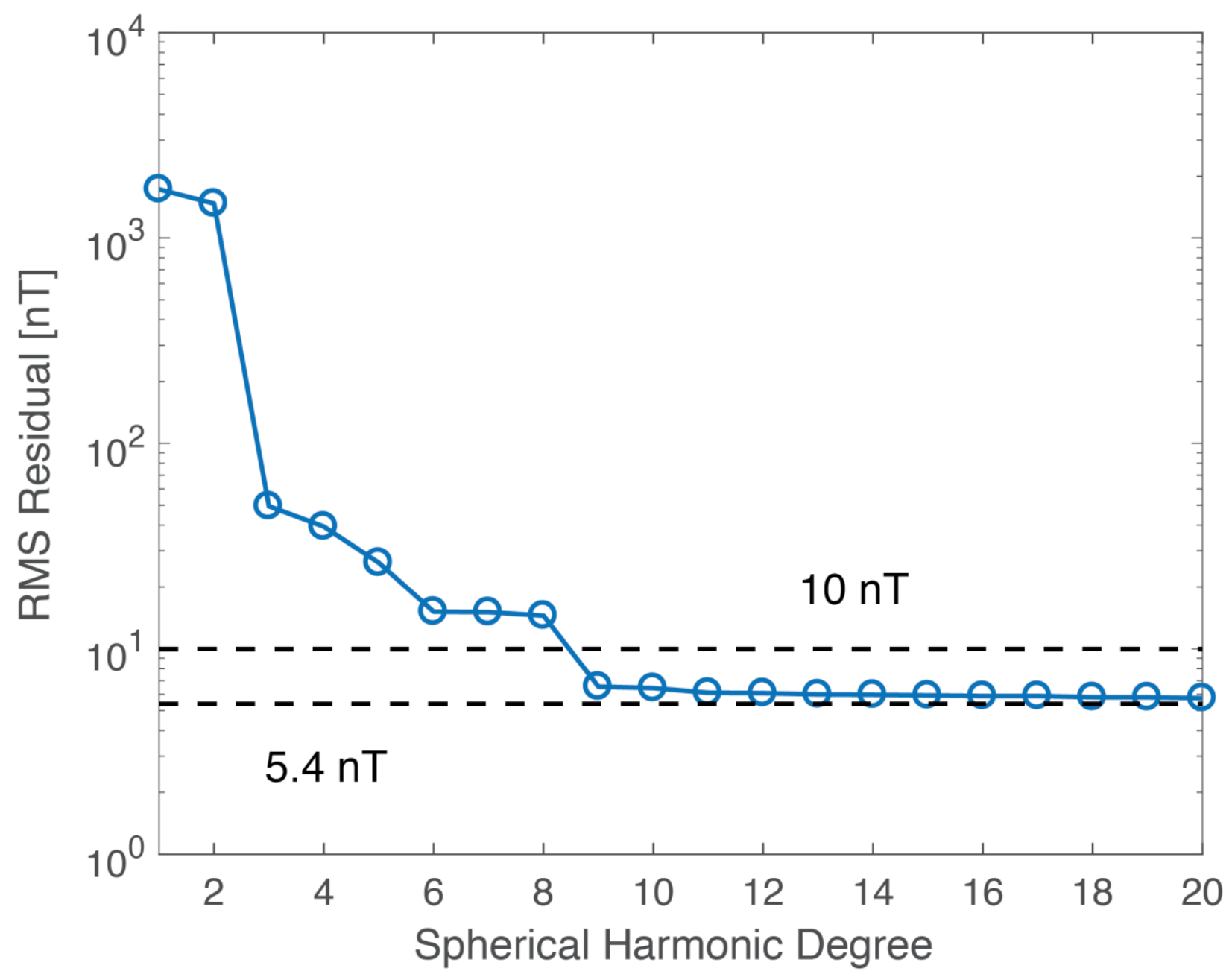

Fig. S5. Root-mean-square (RMS) residual as a function of maximum truncation degree in un-regularized inversion of Saturn's internal magnetic field from Cassini Grand Finale data. A model with Gauss coefficients up to at least spherical harmonic degree 9 is required by the data. 


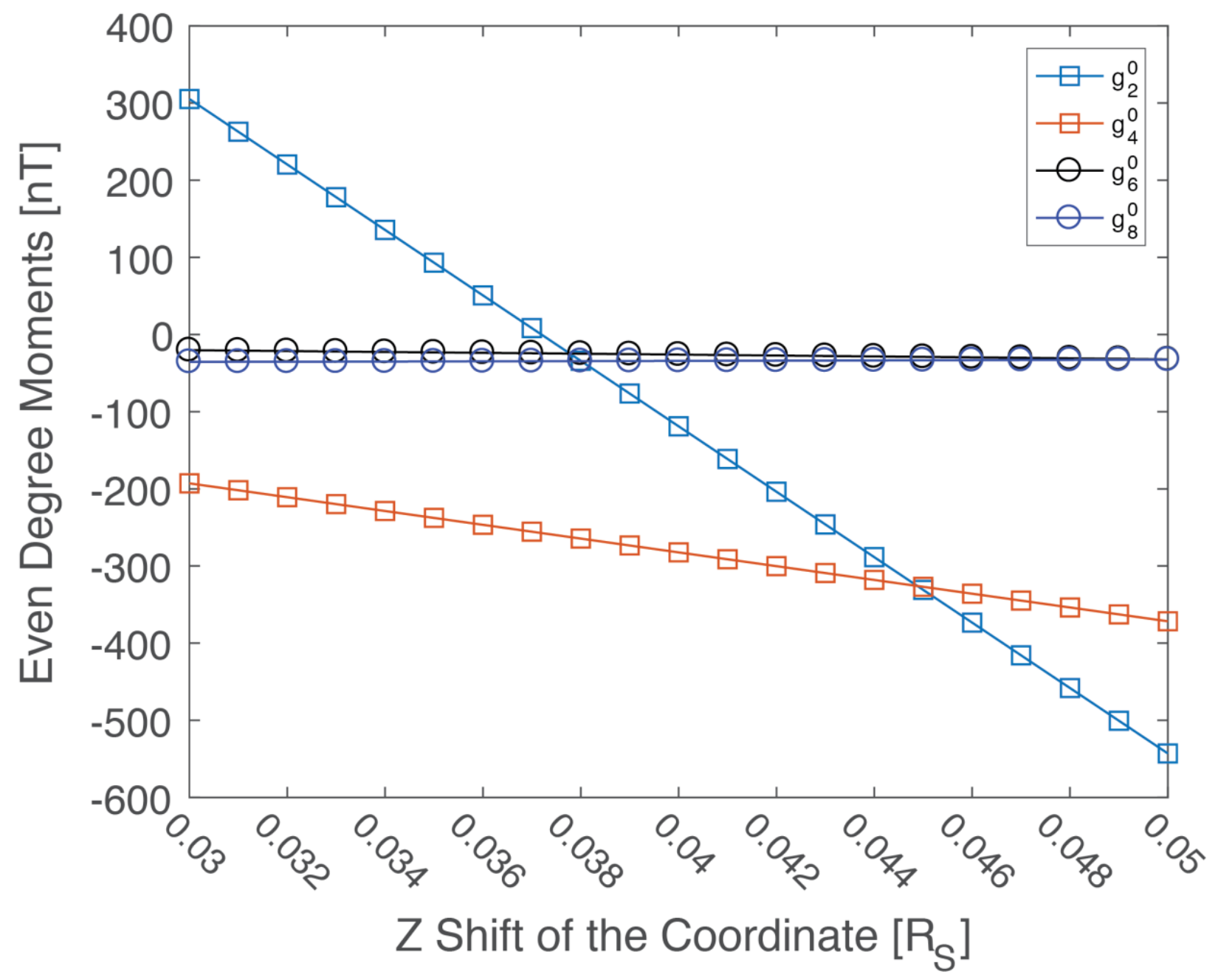

Fig. S6. Gauss coefficients of Saturn's internal magnetic field in Z-shifted coordinates. Only the quadrupole coefficients $\mathrm{g}_{2}^{0}$ becomes zero in a coordinate shifted northward by about $0.037 \mathrm{Rs}$, all other even-degree moments $\mathrm{g}_{4}^{0}, \mathrm{~g}_{6}^{0}, \mathrm{~g}_{8}^{0}$ remain non-zero. In addition, $\mathrm{g}_{4}^{0}$ becomes more than an order of magnitude larger in these shifted coordinates compared to that in the Saturn-centered coordinates. 

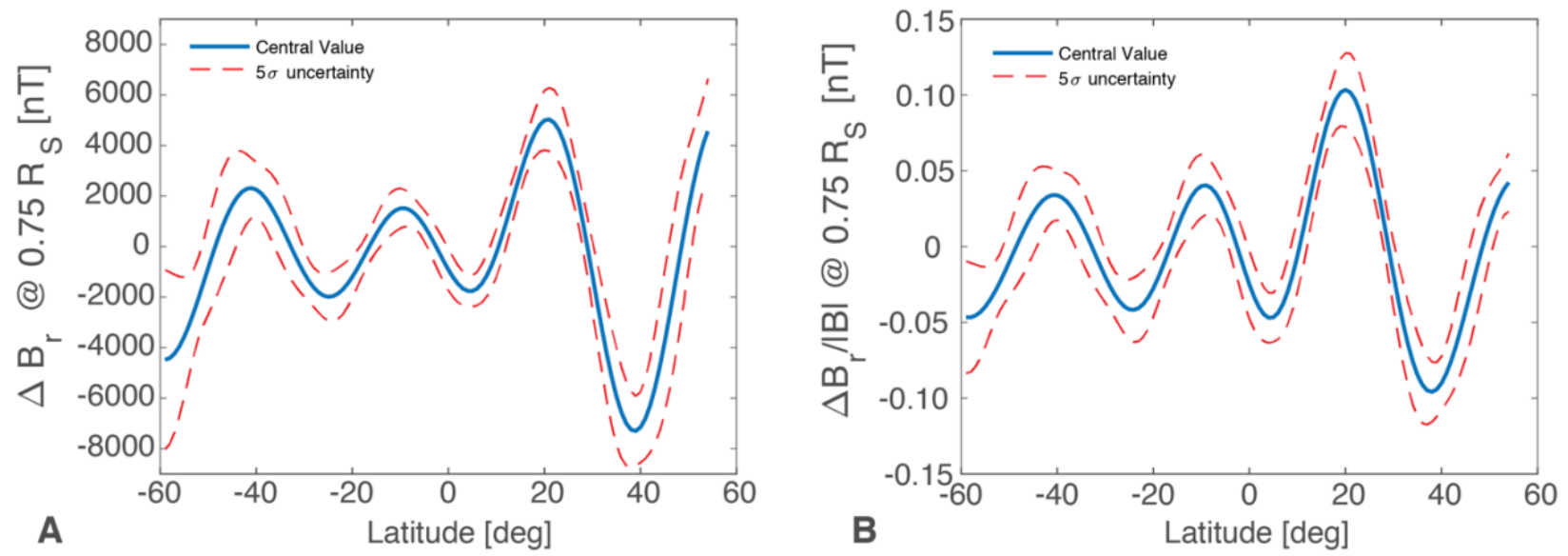

Fig. S7. Small-scale axisymmetric magnetic field of Saturn at $0.75 \boldsymbol{R}_{\boldsymbol{s}}$. Panel A shows $\Delta B_{r}$ while panel B shows $\Delta B_{r} /|B|$. The central value of $\Delta B_{r}$ is computed using the central values of the degree 4 to 11 Gauss coefficients of the Cassini 11 model and value of $|B|=\sqrt{B_{r}^{2}+B_{\theta}^{2}}$ is computed using the central values of the degree 1 to 3 Gauss coefficients of the Cassini 11 model. The uncertainties are computed using the full covariance matrix up to degree 14. The small-scale magnetic perturbations are on the order of 5-10\% of the local background field. 


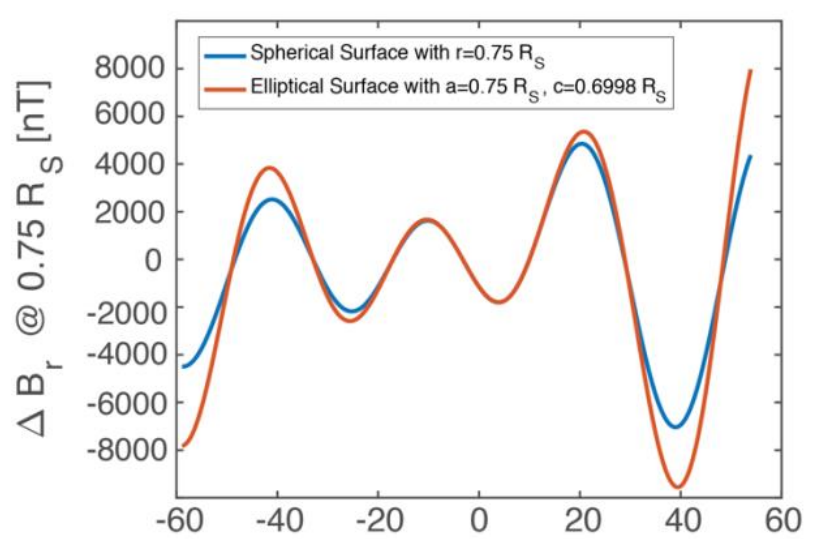

A Latitude [deg]

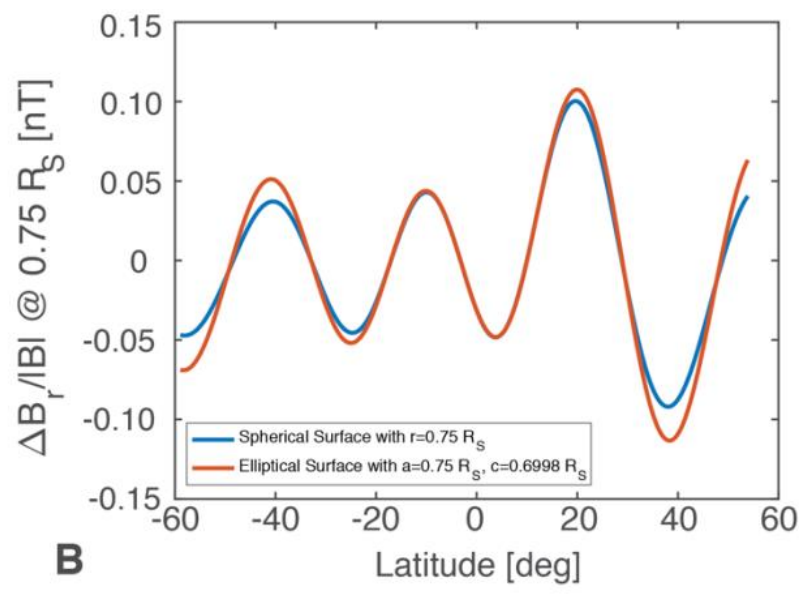

Fig. S8. Small-scale axisymmetric magnetic field of Saturn on a spherical surface with radius $0.75 R_{S}$ and on a dynamically flattened elliptical surface with equatorial radius $0.75 R_{S}$ and polar radius $0.6998 \boldsymbol{R}_{\boldsymbol{S}}$. Panel A shows $\Delta B_{r}$ while panel B shows $\Delta B_{r} /|B|$. The central value of $\Delta B_{r}$ is computed using the central values of the degree 4 to 11 Gauss coefficients of the Cassini 11 model and the value of $|B|=\sqrt{B_{r}^{2}+B_{\theta}^{2}}$ is computed using the central values of the degree 1 to 3 Gauss coefficients of the Cassini 11 model. It can be seen that the small-scale magnetic field are broadly similar on the spherical and dynamically flattened elliptical surface. 


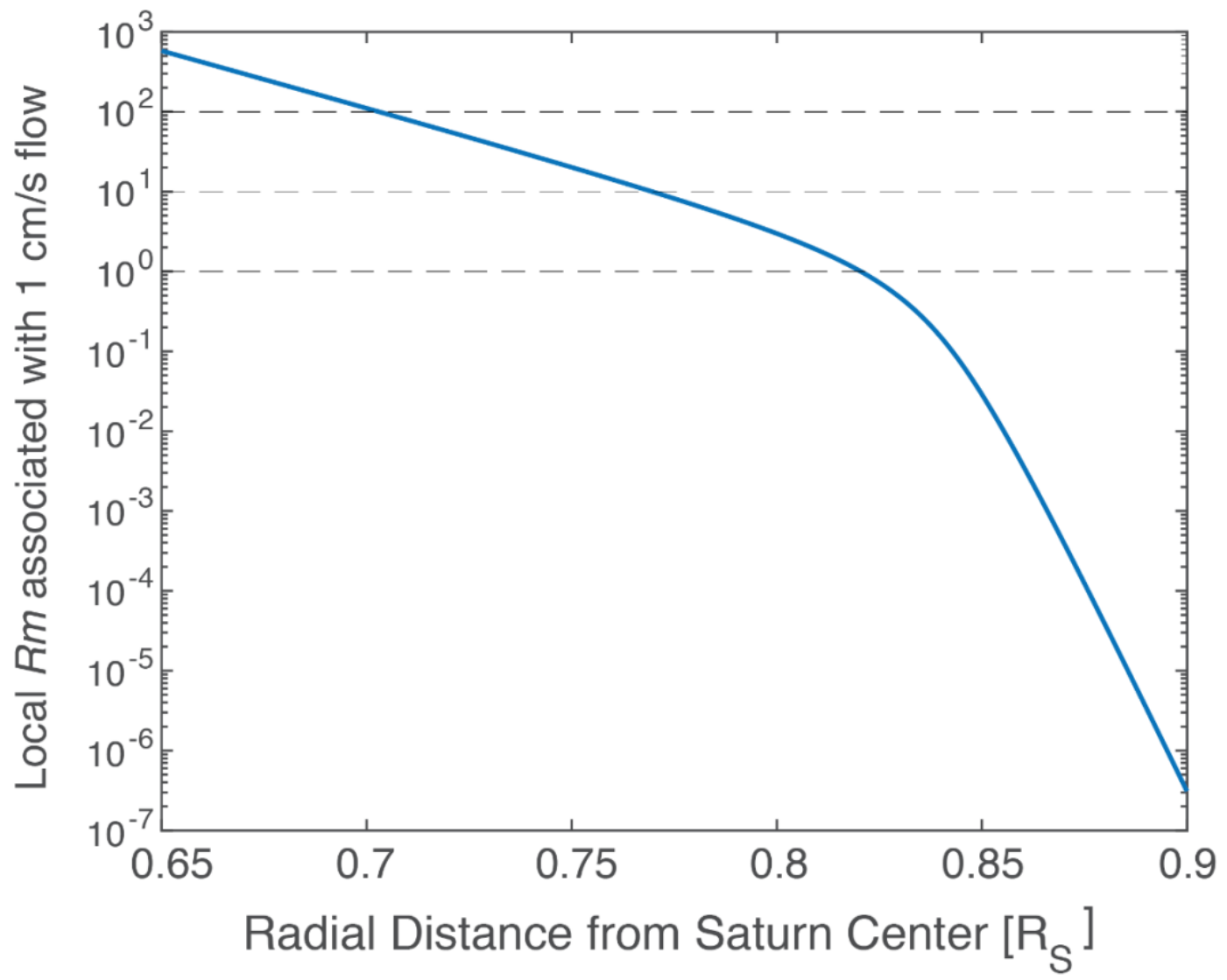

Fig. S9. Local magnetic Reynolds number inside Saturn assuming $1 \mathrm{~cm} / \mathrm{s}$ flow and the electrical conductivity profile of (59). 


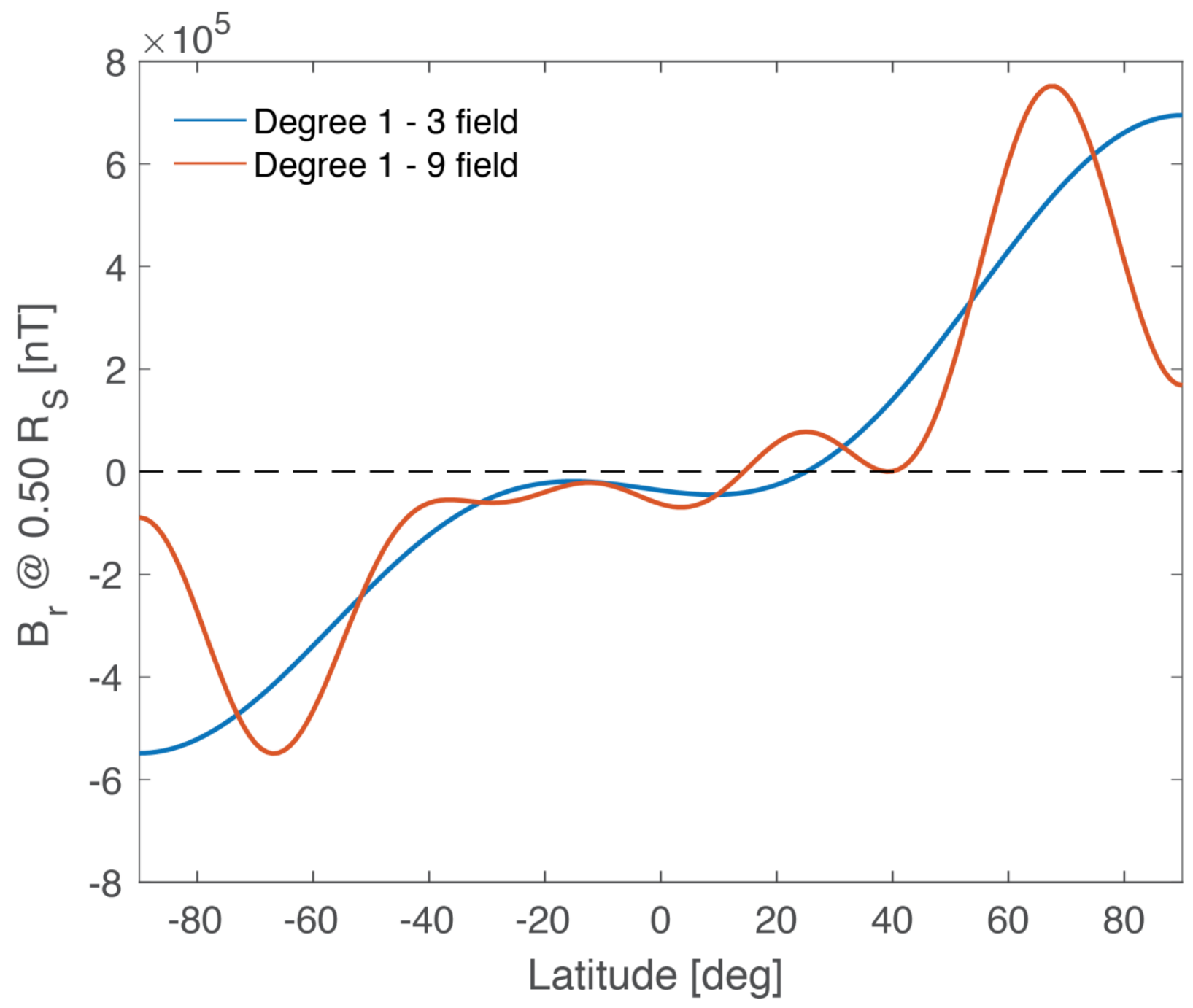

Fig. S10. Saturn's internal magnetic field evaluated at $0.50 R_{S}$. The blue profile is computed from the degree 1 to degree 3 Gauss coefficients while the red profile is computed from the degree 1 to degree 9 Gauss coefficients of the Cassini 11 model (Table 1). 


\begin{tabular}{|l|c|c|}
\hline & Un-regularized degree 3 [nT] & Un-regularized degree 6 [nT] \\
\hline $\mathrm{g}_{1}{ }^{0}$ & 21122 & 21156 \\
\hline $\mathrm{g}_{2}{ }^{0}$ & 1524 & 1591 \\
\hline $\mathrm{g}_{3}{ }^{0}$ & 2218 & 2296 \\
\hline $\mathrm{g}_{4}{ }^{0}$ & & 115 \\
\hline $\mathrm{g}_{5}{ }^{0}$ & & 74 \\
\hline $\mathrm{g}_{6}{ }^{0}$ & & 49 \\
\hline $\begin{array}{l}\text { RMS } \\
\text { Residual }\end{array}$ & 49.4 & 15.2 \\
\hline
\end{tabular}

Table S1. Gauss Coefficients of unregularized internal field models with different truncation 5 degree. 


$\left.\begin{array}{|l|c|c|c|c|}\hline & \begin{array}{l}\text { Inner } \\ \text { edge } \\ a\left[R_{S}\right]\end{array} & \text { Outer edge } & \text { Half thickness } & \mu_{0} I \\ {[n T]}\end{array}\right]$

Table S2. Coefficients of the magnetodisk field model derived for each orbit. 